\title{
SHARP INTEGRAL INEQUALITIES FOR HARMONIC FUNCTIONS
}

\author{
FENGBO HANG, XIAODONG WANG, AND XIAODONG YAN
}

\begin{abstract}
Motivated by Carleman's proof of isoperimetric inequality in the plane, we study some sharp integral inequalities for harmonic functions on the upper halfspace. We also derive the regularity for nonnegative solutions of the associated integral system and some Liouville type theorems.
\end{abstract}

\section{INTRODUCTION}

The classical isoperimetric inequality in the plane states that for any bounded domain with area $A$ and boundary length $L$ we have

$$
4 \pi A \leq L^{2}
$$

and equality holds if and only if the domain is a disk. Inequality (1.1) remains true for bounded domains in a simply connected surface with nonpositive curvature. Among the proofs of this fact the one due to Carleman $[\mathrm{C}$ is particularly interesting. Indeed, let $\left(M^{2}, g\right)$ be any simply connected compact surface with boundary and nonpositive curvature, it follows from Riemann mapping theorem that $\left(M^{2}, g\right)$ is isometric to $\left(\bar{B}_{1}^{2}, e^{2 w} g_{\mathbb{R}^{2}}\right)$, here $B_{1}^{2}$ is the two dimensional open unit disk and $g_{\mathbb{R}^{2}}$ is the Euclidean metric on $\mathbb{R}^{2}$. The nonpositivity of curvature implies that $w$ is a subharmonic function. Let $u$ be the harmonic function on $B_{1}$ with the same boundary value as $w$, then $w \leq u$. In $[\mathrm{C}$ it was proved that for any smooth harmonic function on $\bar{B}_{1}^{2}$ we have

$$
\int_{B_{1}} e^{2 u} d x \leq \frac{1}{4 \pi}\left(\int_{S^{1}} e^{u} d \theta\right)^{2}
$$

and equality holds if and only if $u(x)=c$ or $-2 \log \left|x-x_{0}\right|+c$ for some $x_{0} \in \mathbb{R}^{2} \backslash \bar{B}_{1}$ and constant $c$. We may ask for natural generalizations to higher dimensions. Without an analog of the Riemann mapping theorem, we may start with a metric $g=\rho^{\frac{4}{n-2}} g_{\mathbb{R}^{n}}$ on $\bar{B}_{1}^{n}$ with nonpositive scalar curvature, here $n \geq 3, B_{1}^{n}$ is the open unit ball in $\mathbb{R}^{n}$ and $g_{\mathbb{R}^{n}}$ is the Euclidean metric on $\mathbb{R}^{n}$. It follows that $\rho$ is a subharmonic function. Under the metric $g$ the volume of $\bar{B}_{1}$ is equal to $\int_{B_{1}} \rho^{\frac{2 n}{n-2}} d x$ and the area of $\partial B_{1}$ is equal to $\int_{\partial B_{1}} \rho^{\frac{2(n-1)}{n-2}} d S$. We would like to know whether the inequality

$$
\int_{B_{1}} \rho^{\frac{2 n}{n-2}} d x \leq n^{-\frac{n}{n-1}} \omega_{n}^{-\frac{1}{n-1}}\left(\int_{\partial B_{1}} \rho^{\frac{2(n-1)}{n-2}} d S\right)^{\frac{n}{n-1}}
$$

is still true. Here $\omega_{n}$ is the Euclidean volume of the unit ball in $\mathbb{R}^{n}$. Since $\rho$ is bounded from above by the harmonic function with the same boundary value, we 
only need to know whether the inequality

$$
|u|_{L^{\frac{2 n}{n-2}}\left(B_{1}\right)} \leq n^{-\frac{n-2}{2(n-1)}} \omega_{n}^{-\frac{n-2}{2 n(n-1)}}|u|_{L^{\frac{2(n-1)}{n-2}}\left(\partial B_{1}\right)}
$$

is true for every smooth harmonic function $u$ on $\bar{B}_{1}^{n}$. The answer to this question is affirmative and the inequality may be proved by subcritical approximation (see [HWY]). However, for future purpose it seems helpful to transfer this problem to upper halfspace and derive some Liouville type results. Indeed, assume $u$ is a positive harmonic function on $\bar{B}_{1}$, let $e_{n}=(0, \cdots, 0,1)$ and $\phi$ be the Mobius transformation given by

$$
\phi(x)=\frac{x+\frac{e_{n}}{2}}{\left|x+\frac{e_{n}}{2}\right|^{2}}-e_{n}
$$

Then $\phi\left(\mathbb{R}_{+}^{n}\right)=B_{1}$ and

$$
\phi^{*} g_{\mathbb{R}^{n}}=\frac{1}{\left|x+\frac{e_{n}}{2}\right|^{4}} \sum_{i=1}^{n} d x_{i} \otimes d x_{i}
$$

Here $\mathbb{R}_{+}^{n}=\left\{x \in \mathbb{R}^{n}: x_{n}>0\right\}$. Let

$$
v(x)=\frac{1}{\left|x+\frac{e_{n}}{2}\right|^{n-2}} u\left(\frac{x+\frac{e_{n}}{2}}{\left|x+\frac{e_{n}}{2}\right|^{2}}-e_{n}\right),
$$

then $\phi^{*}\left(u^{\frac{4}{n-2}} g_{\mathbb{R}^{n}}\right)=v^{\frac{4}{n-2}} g_{\mathbb{R}^{n}}$. The inequality (1.3) becomes

$$
|v|_{L^{\frac{2 n}{n-2}}\left(\mathbb{R}_{+}^{n}\right)} \leq n^{-\frac{n-2}{2(n-1)}} \omega_{n}^{-\frac{n-2}{2 n(n-1)}}|v|_{L^{\frac{2(n-1)}{n-2}}\left(\mathbb{R}^{n-1}\right)} .
$$

Note that since $v$ is the Poission integral of $\left.v\right|_{\mathbb{R}^{n-1}}$, inequality (1.4) follows from Theorem 1.1 below. To state the results, let us fix some notations. For convenience, we use $x, y, \cdots$ for points in $\mathbb{R}^{n}$ and $\xi, \zeta, \cdots$ for points in $\mathbb{R}^{n-1}=\left\{x \in \mathbb{R}^{n}: x_{n}=0\right\}$. For $x \in \mathbb{R}^{n}$, we let $x^{\prime}=\left(x_{1}, \cdots, x_{n-1}\right), x=\left(x^{\prime}, x_{n}\right)$. The Poission kernel for the upper half space is given by (see [S, p61])

$$
P(x, \xi)=\frac{2}{n \omega_{n}} \frac{x_{n}}{\left(\left|x^{\prime}-\xi\right|^{2}+x_{n}^{2}\right)^{n / 2}} \quad \text { for } x \in \mathbb{R}_{+}^{n}, \xi \in \mathbb{R}^{n-1} .
$$

Given a function $f$ defined on $\mathbb{R}^{n-1}$, let

$$
(P f)(x)=\int_{\mathbb{R}^{n-1}} P(x, \xi) f(\xi) d \xi \quad \text { for } x \in \mathbb{R}_{+}^{n} .
$$

We have the following sharp inequality for $P$ (see Theorem 4.1):

Theorem 1.1. Assume $n \geq 3$, then for any $f \in L^{\frac{2(n-1)}{n-2}}\left(\mathbb{R}^{n-1}\right)$,

$$
|P f|_{L^{\frac{2 n}{n-2}}\left(\mathbb{R}_{+}^{n}\right)} \leq n^{-\frac{n-2}{2(n-1)}} \omega_{n}^{-\frac{n-2}{2 n(n-1)}}|f|_{L^{\frac{2(n-1)}{n-2}}\left(\mathbb{R}^{n-1}\right)} .
$$

Moreover, equality holds if and only if $f(\xi)=\frac{c}{\left(\lambda^{2}+\left|\xi-\xi_{0}\right|^{2}\right)^{\frac{n-2}{2}}}$ for some constant $c$, positive constant $\lambda$ and $\xi_{0} \in \mathbb{R}^{n-1}$. 
If we look at the variational problem

$$
c_{n}=\sup \left\{|P f|_{L^{\frac{2 n}{n-2}}\left(\mathbb{R}_{+}^{n}\right)}: f \in L^{\frac{2(n-1)}{n-2}}\left(\mathbb{R}^{n-1}\right),|f|_{L^{\frac{2(n-1)}{n-2}}\left(\mathbb{R}^{n-1}\right)}=1\right\} .
$$

Then any nonnegative critical function $f$, after scaling must satisfy

$$
f(\xi)^{\frac{n}{n-2}}=\int_{\mathbb{R}_{+}^{n}} P(x, \xi)(P f)(x)^{\frac{n+2}{n-2}} d x .
$$

We have the following Liouville type theorem (see Proposition 6.1) which is in the same spirit as those in GNN, CGS, CLO2, L.

Theorem 1.2. Assume $n \geq 3, f \in L_{l o c}^{\frac{2(n-1)}{n-2}}\left(\mathbb{R}^{n-1}\right)$ is nonnegative, not identically zero and it satisfies (1.7), then for some $\lambda>0$ and $\xi_{0} \in \mathbb{R}^{n-1}$,

$$
f(\xi)=c(n)\left(\frac{\lambda}{\lambda^{2}+\left|\xi-\xi_{0}\right|^{2}}\right)^{\frac{n-2}{2}} .
$$

We note that the condition $f \in L_{l o c}^{\frac{2(n-1)}{n-2}}\left(\mathbb{R}^{n-1}\right)$ can not be dropped since $c(n)|\xi|^{-\frac{n-2}{2}}$ is a singular solution for (1.7). During the process of identifying maximizing functions in Theorem 1.1 and the critical functions in Theorem 1.2, we establish the following interesting fact (see Proposition 4.1):

Proposition 1.1. Let $n \geq 2$, $u$ be a function on $\mathbb{R}^{n}$ which is radial with respect to the origin, $0<u(x)<\infty$ for $x \neq 0, e_{1}=(1,0, \cdots, 0), \alpha \in \mathbb{R}, \alpha \neq 0$. If $v(x)=|x|^{\alpha} u\left(\frac{x}{|x|^{2}}-e_{1}\right)$ is radial with respect to some point, then either $u(x)=$ $\left(c_{1}|x|^{2}+c_{2}\right)^{\alpha / 2}$ for some $c_{1} \geq 0, c_{2}>0$ or

$$
u(x)=\left\{\begin{array}{l}
c_{1}|x|^{\alpha}, \text { if } x \neq 0, \\
c_{2}, \text { if } x=0,
\end{array}\right.
$$

for some $c_{1}>0$ and $c_{2}$, an arbitrary number.

There are similar statements for the cases $\alpha=0$ or $n=1$ (see Remark 4.1 and Proposition 4.2). The crucial point of Proposition 1.1 is that we do not need any regularity assumption on the function $u$. This is very convenient when the regularity of extremal functions are hard to get apriorly. The radial symmetry property of function may come from symmetrization arguments or the method of moving planes etc. For example, Proposition 1.1 gives another way to determine the maximizing functions for those cases of Hardy-Littlewood-Sobolev inequalities studied in [Li2, section III]. The formulation of Proposition 1.1] is motivated from previous works in [CL, O, CLO2, section 3] and [CLO3, section 6]. It is worth pointing out that Proposition 1.1 is the fact for method of moving planes which corresponds to the fact [LZ, lemma 2.5] or [L, lemma 5.8] for the method of moving spheres, a variant of the method of moving planes.

According to Proposition 2.1 below, for $n \geq 2$ and $1<p<\infty$ the operator

$$
P: L^{p}\left(\mathbb{R}^{n-1}\right) \rightarrow L^{\frac{n p}{n-1}}\left(\mathbb{R}_{+}^{n}\right): f \mapsto P f
$$


is always a bounded linear map. From the analytical point view it is interesting to consider the variational problem

$$
c_{n, p}=\sup \left\{|P f|_{L^{\frac{n p}{n-1}}\left(\mathbb{R}_{+}^{n}\right)}: f \in L^{p}\left(\mathbb{R}^{n-1}\right),|f|_{L^{p}\left(\mathbb{R}^{n-1}\right)}=1\right\}
$$

for all such $p$ 's. Fix $1<p<\infty$, for a function $f$ defined on $\mathbb{R}^{n-1}, \lambda>0$ and $\zeta \in \mathbb{R}^{n-1}$, we write

$$
f^{\lambda, \zeta}(\xi)=\lambda^{-\frac{n-1}{p}} f\left(\frac{\xi-\zeta}{\lambda}\right) \quad \text { for } \xi \in \mathbb{R}^{n-1} .
$$

Then we have (see Theorem 3.1 and Theorem 4.1):

Theorem 1.3. Given $n \geq 2$ and $1<p<\infty$.

- Let $f_{i}$ be a maximizing sequence of functions for (1.8), then after passing to a subsequence there exists $\lambda_{i}>0$ and $\zeta_{i} \in \mathbb{R}^{n-1}$ such that $f_{i}^{\lambda_{i}, \zeta_{i}} \rightarrow f$ in $L^{p}\left(\mathbb{R}^{n-1}\right)$. In particular, there exists at least one maximizing function.

- After multiplying by a nonzero constant, every maximizer $f$ of (1.8) is nonnegative, radial symmetric with respect to some point, strictly decreasing in the radial direction and it satisfies

$$
f(\xi)^{p-1}=\int_{\mathbb{R}_{+}^{n}} P(x, \xi)(P f)(x)^{\frac{n p}{n-1}-1} d x .
$$

- If $n \geq 3$ and $p=\frac{2(n-1)}{n-2}$, then any maximizer of (1.8) must be of the form

$$
f(\xi)= \pm c(n)\left(\frac{\lambda}{\lambda^{2}+\left|\xi-\xi_{0}\right|^{2}}\right)^{\frac{n-2}{2}}
$$

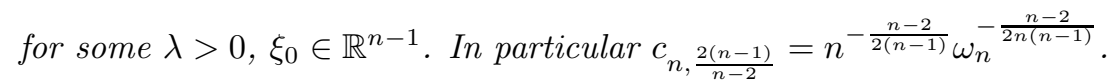

- If $n \geq 3$ and $p=\frac{2(n-1)}{n}$, then any maximizer of (1.8) must be of the form

$$
f(\xi)= \pm c(n)\left(\frac{\lambda}{\lambda^{2}+\left|\xi-\xi_{0}\right|^{2}}\right)^{n / 2}
$$

for some $\lambda>0, \xi_{0} \in \mathbb{R}^{n-1}$. In particular

$$
c_{n, \frac{2(n-1)}{n}}=\frac{1}{\sqrt{2(n-2) \sqrt[4]{\pi}}}\left(\frac{(n-2) !}{\Gamma\left(\frac{n-1}{2}\right)}\right)^{\frac{1}{2(n-1)}} .
$$

It is interesting that the problem considered here demonstrates very similar structures to the sharp Hardy-Littlewood-Sobolev inequalities studied in Li2. Besides above properties of maximizing functions, we know they are smooth. This is a nontrivial fact since it does not follow from the usual bootstrap method. Indeed, we know all the nonnegative critical functions of (1.8) are smooth and radial symmetric with respect to some point (see Theorem 5.1 and Theorem 6.1). More precisely we have

Theorem 1.4. Given $n \geq 2$ and $1<p<\infty$. If $f \in L^{p}\left(\mathbb{R}^{n-1}\right)$ is nonnegative, not identically zero and it satisfies (1.9), then $f \in C^{\infty}\left(\mathbb{R}^{n-1}\right)$, moreover it is radial symmetric with respect to some point and strictly decreasing along the radial direction. 
In Section 2 below, we will collect some basic estimates for Poission integrals and show the operator $P$ is bounded in suitable Lebesgue spaces and Lorentz spaces. In Section 3, we apply the general frame of concentration compactness principle ([Lion] to show that every maximizing sequence of (1.8), after scaling and translation, must converge strongly. In Section 4, following Lieb we use the method of symmetrization based on the Riesz rearrangement inequalities ([LiL, section 3.7]) and its strong form ([Li1] to show that all maximizing functions must be radial and give another approach to the existence of maximizing functions. In Section 5 we use the method in $\mathrm{Hn}$ to deduce the regularity of all nonnegative critical functions. Indeed what we will prove is a local regularity result. These results are similar in nature to those proved in $\mathrm{ChL}, \mathrm{L}$. In Section 6 we use the integral version of the method of moving planes (GNN]), which was discovered in [CLO2, to deduce the symmetry property of the nonnegative critical functions. Here we will need some ideas from $\mathrm{Hn}$ again.

Acknowledgment: The research of F. Hang is supported by National Science Foundation Grant DMS-0501050 and a Sloan Research Fellowship. The research of X. Wang is supported by National Science Foundation Grant DMS-0505645. The research of X. Yan is supported by National Science Foundation Grant DMS0401048 and an IRGP grant from Michigan State University.

\section{Basic inequalities for Poission integrals}

The main aim of this section is to record some basic estimates associated with Poission kernel and harmonic extensions which we will use freely later. For $x_{0} \in \mathbb{R}^{n}$ and $r>0$, we write

$$
B_{r}^{n}\left(x_{0}\right)=\left\{x \in \mathbb{R}^{n}:\left|x-x_{0}\right|<r\right\}, \quad B_{r}^{n}=B_{r}^{n}(0), \quad B_{r}^{+}=B_{r}^{n} \cap \mathbb{R}_{+}^{n}
$$

and $\bar{B}_{r}^{n}\left(x_{0}\right)$ to mean the closure of $B_{r}^{n}\left(x_{0}\right)$. Assume $n \geq 2$. For $t>0, \xi \in \mathbb{R}^{n-1}$, we write

Clearly we have

$$
P_{t}(\xi)=\frac{2}{n \omega_{n}} \frac{t}{\left(|\xi|^{2}+t^{2}\right)^{n / 2}} .
$$

- $P(x, \xi)=P_{x_{n}}\left(x^{\prime}-\xi\right)$ for $x \in \mathbb{R}_{+}^{n}, \xi \in \mathbb{R}^{n-1}$.

- $(P f)(x)=\left(P_{x_{n}} * f\right)\left(x^{\prime}\right)$ for $x \in \mathbb{R}_{+}^{n}$.

- $\left|P_{t}\right|_{L^{1}\left(\mathbb{R}^{n-1}\right)}=1,\left|P_{t}\right|_{L^{\infty}\left(\mathbb{R}^{n-1}\right)}=\frac{2}{n \omega_{n}} \frac{1}{t^{n-1}}$.

- $\left|P_{t}\right|_{L^{p}\left(\mathbb{R}^{n-1}\right)}=c(n, p) t^{-\frac{(n-1)(p-1)}{p}}$ for $\frac{n-1}{n}<p \leq \infty$.

Recall if $X$ is a measure space, $p>0$ and $u$ is a measurable function on $X$, then

$$
|u|_{L_{W}^{p}(X)}=\sup _{t>0} t|| u|>t|^{1 / p} .
$$

The space $L_{W}^{p}(X)=\left\{u: u\right.$ is measurable and $\left.|u|_{L_{W}^{p}(X)}<\infty\right\}$. More generally, for any $0<p<\infty$ and $0<q \leq \infty$, we have the Lorentz norm $|\cdot|_{L^{p, q}(X)}$ and Lorentz space $L^{p, q}(X)$ (see [SW, p188]). $L_{W}^{p}(X)=L^{p, \infty}(X)$ is a special case of such spaces.

Proposition 2.1. We have

$$
|P f|_{L_{W}^{\frac{n}{n-1}}\left(\mathbb{R}_{+}^{n}\right)} \leq c(n)|f|_{L^{1}\left(\mathbb{R}^{n-1}\right)}
$$


and

$$
|P f|_{L^{\frac{n p}{n-1}}\left(\mathbb{R}_{+}^{n}\right)} \leq c(n, p)|f|_{L^{p}\left(\mathbb{R}^{n-1}\right)}
$$

for $1<p \leq \infty$. Moreover for $1<p<\infty$ we have

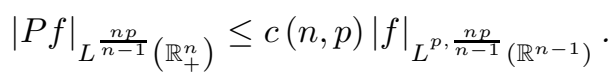

Proof. We only need to prove the weak type estimate. The strong estimate follows from Marcinkiewicz interpolation theorem (see [SW, p197]) and the basic fact $|P f|_{L^{\infty}\left(\mathbb{R}_{+}^{n}\right)} \leq|f|_{L^{\infty}\left(\mathbb{R}^{n-1}\right)}$. To prove the weak type estimate, we may assume $f \geq 0$ and $|f|_{L^{1}\left(\mathbb{R}^{n-1}\right)}=1$. First we observe that $(P f)(x) \leq \frac{c(n)}{x_{n}^{n-1}}$ for $x \in \mathbb{R}_{+}^{n}$ and

$$
\int_{x \in \mathbb{R}_{+}^{n}, 0<x_{n}<a}(P f)(x) d x=\int_{\mathbb{R}^{n-1}} d \xi\left(f(\xi) \int_{0}^{a} d x_{n} \int_{\mathbb{R}^{n-1}} P(x, \xi) d x^{\prime}\right)=a
$$

for $a>0$. Hence for $t>0$,

$$
\begin{aligned}
|P f>t| & =\left|\left\{x \in \mathbb{R}_{+}^{n}: 0<x_{n}<c(n) t^{-\frac{1}{n-1}},(P f)(x)>t\right\}\right| \\
& \leq \frac{1}{t} \int_{0<x_{n}<c(n) t^{-\frac{1}{n-1}, x^{\prime} \in \mathbb{R}^{n-1}}}(P f)(x) d x=c(n) t^{-\frac{n}{n-1}} .
\end{aligned}
$$

The weak type inequality follows.

Later we will also need some elementary estimates for the harmonic extensions. They are listed below.

- For $1 \leq p \leq q \leq \infty$, we have

$$
\left|P_{t} * f\right|_{L^{q}\left(\mathbb{R}^{n-1}\right)} \leq c(n, p, q) t^{-(n-1)\left(\frac{1}{p}-\frac{1}{q}\right)}|f|_{L^{p}\left(\mathbb{R}^{n-1}\right)} .
$$

- Assume $f(\xi)=0$ for $|\xi| \geq R$, then we have

$$
\left|\left(P_{t} * f\right)(\xi)\right| \leq \frac{c(n) t}{\left[\left((|\xi|-R)^{+}\right)^{2}+t^{2}\right]^{n / 2}}|f|_{L^{1}\left(\mathbb{R}^{n-1}\right)} .
$$

- Assume $f(\xi)=0$ for $|\xi|<R, 1 \leq p \leq \infty$, then we have

$$
\left|P_{t} * f\right|_{L^{\infty}\left(B_{R / 2}^{n-1}\right)} \leq c(n, p) t R^{-\frac{n-1}{p}-1}|f|_{L^{p}\left(\mathbb{R}^{n-1}\right)}
$$

and

$$
|P f|_{L^{\infty}\left(B_{R / 2}^{+}\right)} \leq c(n, p) R^{-\frac{n-1}{p}}|f|_{L^{p}\left(\mathbb{R}^{n-1}\right)} .
$$

For $t>0, \xi \in \mathbb{R}^{n-1}$, let

$$
Q_{t}(\xi)=P_{t}(\xi) \cdot \frac{|\xi|}{t}=\frac{2}{n \omega_{n}} \frac{|\xi|}{\left(|\xi|^{2}+t^{2}\right)^{n / 2}},
$$

then

- $\left|Q_{t}\right|_{L^{p}\left(\mathbb{R}^{n-1}\right)}=c(n, p) t^{-\frac{(n-1)(p-1)}{p}}$ for $1<p \leq \infty$. 
- Let $\varphi \in L^{\infty}\left(\mathbb{R}^{n-1}\right) \cap \operatorname{Lip}\left(\mathbb{R}^{n-1}\right)$, then

$$
\left|P_{t} *(\varphi f)-\varphi\left(P_{t} * f\right)\right| \leq[\varphi]_{\operatorname{Lip}\left(\mathbb{R}^{n-1}\right)} t Q_{t} * f .
$$

In particular, it follows from Hausdorff-Young's inequality that

$$
\begin{aligned}
& \left|P_{t} *(\varphi f)-\varphi\left(P_{t} * f\right)\right|_{L^{q}\left(\mathbb{R}^{n-1}\right)} \\
& \leq c(n, p, q)[\varphi]_{\operatorname{Lip}\left(\mathbb{R}^{n-1}\right)} t^{1-(n-1)\left(\frac{1}{p}-\frac{1}{q}\right)}|f|_{L^{p}\left(\mathbb{R}^{n-1}\right)}
\end{aligned}
$$

for $1 \leq p<q \leq \infty$.

As a simple application of these estimates, we derive the following compactness result.

Corollary 2.1. For $1 \leq p<\infty, 1 \leq q<\frac{n p}{n-1}$, the operator

$$
P: L^{p}\left(\mathbb{R}^{n-1}\right) \rightarrow L_{l o c}^{q}\left(\overline{\mathbb{R}_{+}^{n}}\right)
$$

is compact.

Proof. Assume $f_{i} \in L^{p}\left(\mathbb{R}^{n-1}\right)$ such that $\left|f_{i}\right|_{L^{p}\left(\mathbb{R}^{n-1}\right)} \leq 1$, it follows that

$$
\left|\left(P f_{i}\right)(x)\right| \leq c(n, p) x_{n}^{-\frac{n-1}{p}} \text { for } x \in \mathbb{R}_{+}^{n} .
$$

By the gradient estimates of harmonic functions, after passing to a subsequence we have $P f_{i} \rightarrow u$ in $C_{l o c}^{\infty}\left(\mathbb{R}_{+}^{n}\right)$. For any $R>0$,

$$
\begin{aligned}
& \left|P f_{i}-P f_{j}\right|_{L^{q}\left(B_{R}^{+}\right)}^{q}\left|P f_{i}-P f_{j}\right|^{q} d x+\int_{x \in B_{R}^{+}, x_{n}<\varepsilon}\left|P f_{i}-P f_{j}\right|^{q} d x \\
& =\int_{x \in B_{R}^{+}, x_{n} \geq \varepsilon}\left|P f_{i}-P f_{j}\right|^{q} d x+c(n, p, q) \varepsilon^{1-(n-1)\left(\frac{q}{p}-1\right)} . \\
& \leq \int_{x \in B_{R}^{+}, x_{n} \geq \varepsilon}
\end{aligned}
$$

Hence

$$
\lim \sup _{i, j \rightarrow \infty}\left|P f_{i}-P f_{j}\right|_{L^{q}\left(B_{R}^{+}\right)}^{q} \leq c(n, p, q) \varepsilon^{1-(n-1)\left(\frac{q}{p}-1\right)} .
$$

Let $\varepsilon \rightarrow 0^{+}$, we see $P f_{i}$ is a Cauchy sequence in $L_{l o c}^{q}\left(\overline{\mathbb{R}_{+}^{n}}\right)$.

Finally we derive a dual statement to Proposition 2.1. Let $u$ be a function on $\mathbb{R}_{+}^{n}$, we write

$$
(T u)(\xi)=\int_{\mathbb{R}_{+}^{n}} P(x, \xi) u(x) d x .
$$

Proposition 2.2. For $1 \leq p<n$ we have

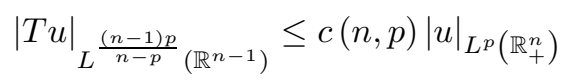

for any $u \in L^{p}\left(\mathbb{R}_{+}^{n}\right)$. 
Proof. We may prove the inequality by a duality argument. Indeed, for any nonnegative functions $u$ on $\mathbb{R}_{+}^{n}$ and $f$ on $\mathbb{R}^{n-1}$ we have

$$
\begin{aligned}
0 & \leq \int_{\mathbb{R}^{n-1}}(T u)(\xi) f(\xi) d \xi=\int_{\mathbb{R}^{n-1}} d \xi \int_{\mathbb{R}_{+}^{n}} P(x, \xi) u(x) f(\xi) d x \\
& =\int_{\mathbb{R}_{+}^{n}}(P f)(x) u(x) d x \leq|P f|_{L^{\frac{p}{p-1}}\left(\mathbb{R}_{+}^{n}\right)}|u|_{L^{p}\left(\mathbb{R}_{+}^{n}\right)} \\
& \leq c(n, p)|u|_{L^{p}\left(\mathbb{R}_{+}^{n}\right)}|f|_{L^{\frac{(n-1) p}{n(p-1)}}\left(\mathbb{R}^{n-1}\right)} .
\end{aligned}
$$

Inequality (2.3) follows. We may also prove such an inequality directly. Indeed, since

$$
|P(\cdot, \xi)|_{L^{\frac{n}{n-1}, \infty}\left(\mathbb{R}_{+}^{n}\right)}=|P(\cdot, 0)|_{L^{\frac{n}{n-1}, \infty}\left(\mathbb{R}_{+}^{n}\right)}=c(n)<\infty,
$$

we see $T: L^{n, 1}\left(\mathbb{R}_{+}^{n}\right) \rightarrow L^{\infty}\left(\mathbb{R}^{n-1}\right)$ is a bounded linear map. On the other hand, for $u \in L^{1}\left(\mathbb{R}_{+}^{n}\right)$, we have

$$
\int_{\mathbb{R}^{n-1}}|(T u)(\xi)| d \xi \leq \int_{\mathbb{R}^{n-1}} d \xi \int_{\mathbb{R}_{+}^{n}} P(x, \xi)|u(x)| d x=\int_{\mathbb{R}_{+}^{n}}|u(x)| d x .
$$

Hence $T: L^{1}\left(\mathbb{R}_{+}^{n}\right) \rightarrow L^{1}\left(\mathbb{R}^{n-1}\right)$ is also bounded. The inequality (2.3) follows from the Marcinkiewicz interpolation theorem.

3. The EXISTENCE OF MAXIMIZING FUnCTIONS FOR SHARP INEQUALITIES BY THE CONCENTRATION COMPACTNESS PRINCIPLE

Assume $n \geq 2$ and $1<p<\infty$. Let $c_{n, p}$ be the sharp constant in (2.1), then $c_{n, p}>0$ and

$$
c_{n, p}^{\frac{n p}{n-1}}=\sup \left\{\int_{\mathbb{R}_{+}^{n}}|P f|^{\frac{n p}{n-1}} d x: f \in L^{p}\left(\mathbb{R}^{n-1}\right),|f|_{L^{p}\left(\mathbb{R}^{n-1}\right)}=1\right\} .
$$

The aim of this section is to show $c_{n, p}^{\frac{n p}{n-1}}$ is attained by some functions. Let $f$ be a function defined on $\mathbb{R}^{n-1}$. For $\lambda>0$ and $\zeta \in \mathbb{R}^{n-1}$ we write $f^{\lambda, \zeta}(\xi)=$ $\lambda^{-\frac{n-1}{p}} f\left(\frac{\xi-\zeta}{\lambda}\right)$ for $\xi \in \mathbb{R}^{n-1}$, then

$$
\left|f^{\lambda, \zeta}\right|_{L^{p}\left(\mathbb{R}^{n-1}\right)}=|f|_{L^{p}\left(\mathbb{R}^{n-1}\right)}, \quad\left|P f^{\lambda, \zeta}\right|_{L^{\frac{n p}{n-1}}\left(\mathbb{R}_{+}^{n}\right)}=|P f|_{L^{\frac{n p}{n-1}}\left(\mathbb{R}_{+}^{n}\right)} .
$$

In particular the variational problem (3.1) has both translation and dilation invariance. The problem fits in the general frame of concentration compactness principle of [Lion. We will apply this principle to prove the following result.

Theorem 3.1. Assume $n \geq 2$ and $1<p<\infty$. Let $f_{i}$ be a maximizing sequence of functions for (3.1), then after passing to a subsequence there exists $\lambda_{i}>0$ and $\zeta_{i} \in \mathbb{R}^{n-1}$ such that $f_{i}^{\lambda_{i}, \zeta_{i}} \rightarrow f$ in $L^{p}\left(\mathbb{R}^{n-1}\right)$. In particular, there exists at least one maximizing function for the variational problem (3.1).

A basic ingredient in the proof of Theorem 3.1 is the following proposition corresponding to [Lion, lemma 2.1].

Proposition 3.1. Assume $n \geq 2,1<p<\infty$ and $f_{i} \in L^{p}\left(\mathbb{R}^{n-1}\right)$ such that $f_{i} \rightarrow f$ in $L^{p}\left(\mathbb{R}^{n-1}\right)$. After passing to a subsequence, assume

$$
\left|f_{i}\right|^{p} d \xi \rightarrow \mu \text { in } \mathcal{M}\left(\mathbb{R}^{n-1}\right), \quad\left|P f_{i}\right|^{\frac{n p}{n-1}} d x \rightarrow \nu \text { in } \mathcal{M}\left(\overline{\mathbb{R}_{+}^{n}}\right) .
$$


Here $\mathcal{M}\left(\mathbb{R}^{n-1}\right)$ denotes the space of all Radon measures on $\mathbb{R}^{n-1}$. Then we have

$$
\left.\nu\right|_{\mathbb{R}_{+}^{n}}=|P f|^{\frac{n p}{n-1}} d x .
$$

Moreover for any Borel set $E \subset \mathbb{R}^{n-1}$,

$$
\nu(E)^{\frac{n-1}{n p}} \leq c_{n, p} \mu(E)^{\frac{1}{p}} .
$$

- There exists a countable set of points $\zeta_{j} \in \mathbb{R}^{n-1}$ such that

$$
\nu=|P f|^{\frac{n p}{n-1}} d x+\sum_{j} \nu_{j} \delta_{\zeta_{j}}, \mu \geq|f|^{p} d \xi+\sum_{j} \mu_{j} \delta_{\zeta_{j}},
$$

here $\mu_{j}=\mu\left(\left\{\zeta_{j}\right\}\right)$ and

$$
\nu_{j}^{\frac{n-1}{n p}} \leq c_{n, p} \mu_{j}^{\frac{1}{p}} .
$$

- If $\nu\left(\mathbb{R}^{n-1}\right)^{\frac{n-1}{n p}} \geq c_{n, p} \mu\left(\mathbb{R}^{n-1}\right)^{\frac{1}{p}}$, then $\nu$ is supported on a single point.

Proof. Without losing of generality, we may assume $\left|f_{i}\right|_{L^{p}\left(\mathbb{R}^{n-1}\right)} \leq 1$. Since

$$
\left|\left(P f_{i}\right)(x)\right| \leq c(n, p) x_{n}^{-\frac{n-1}{p}} \text { for } x \in \mathbb{R}_{+}^{n},
$$

it follows from the gradient estimate of harmonic functions that $P f_{i} \rightarrow P f$ in $C_{\text {loc }}^{\infty}\left(\mathbb{R}_{+}^{n}\right)$. In particular,

$$
\left.\nu\right|_{\mathbb{R}_{+}^{n}}=|P f|^{\frac{n p}{n-1}} d x .
$$

Let $\varphi \in C_{c}^{\infty}\left(\mathbb{R}^{n-1}\right)$ and $\eta \in C_{c}^{\infty}([0, \infty))$ such that $0 \leq \eta \leq 1$, we have

$$
\begin{aligned}
& \left|\varphi\left(x^{\prime}\right) \eta\left(x_{n}\right)\left(P f_{i}\right)(x)\right|_{L^{\frac{n p}{n-1}}\left(\mathbb{R}_{+}^{n}\right)} \\
& \leq\left|\eta\left(x_{n}\right) P\left(\varphi f_{i}\right)(x)\right|_{L^{\frac{n p}{n-1}}\left(\mathbb{R}_{+}^{n}\right)}+\left|\eta\left(x_{n}\right)\left(\varphi\left(x^{\prime}\right)\left(P f_{i}\right)(x)-P\left(\varphi f_{i}\right)(x)\right)\right|_{L^{\frac{n p}{n-1}}\left(\mathbb{R}_{+}^{n}\right)} \\
& \leq c_{n, p}\left|\varphi f_{i}\right|_{L^{p}\left(\mathbb{R}^{n-1}\right)}+c(n, p)|\nabla \varphi|_{L^{\infty}\left(\mathbb{R}^{n-1}\right)}\left(\int_{0}^{\infty} \eta(t)^{\frac{n p}{n-1}} t^{\frac{n p}{n-1}-1} d t\right)^{\frac{n-1}{n p}} .
\end{aligned}
$$

Now fix an $\eta \in C^{\infty}([0, \infty))$ such that $0 \leq \eta \leq 1, \eta(0)=1$ and $\eta(t)=0$ for $t \geq 1$. For $\varepsilon>0$, denote $\eta_{\varepsilon}(t)=\eta(t / \varepsilon)$. Then

$$
\begin{aligned}
& \left|\varphi\left(x^{\prime}\right) \eta_{\varepsilon}\left(x_{n}\right)\left(P f_{i}\right)(x)\right|_{L^{\frac{n p}{n-1}}\left(\mathbb{R}_{+}^{n}\right)} \\
& \leq c_{n, p}\left|\varphi f_{i}\right|_{L^{p}\left(\mathbb{R}^{n-1}\right)}+c(n, p)|\nabla \varphi|_{L^{\infty}\left(\mathbb{R}^{n-1}\right)} \varepsilon .
\end{aligned}
$$

Letting $i \rightarrow \infty$ and then $\varepsilon \rightarrow 0^{+}$, we see

$$
\left(\int_{\mathbb{R}^{n-1}}|\varphi|^{\frac{n p}{n-1}} d \nu\right)^{\frac{n-1}{n p}} \leq c_{n, p}\left(\int_{\mathbb{R}^{n-1}}|\varphi|^{p} d \mu\right)^{1 / p} .
$$

A limit process shows for any Borel function $h$ on $\mathbb{R}^{n-1}$,

$$
\left(\int_{\mathbb{R}^{n-1}}|h|^{\frac{n p}{n-1}} d \nu\right)^{\frac{n-1}{n p}} \leq c_{n, p}\left(\int_{\mathbb{R}^{n-1}}|h|^{p} d \mu\right)^{1 / p} .
$$

This implies for any Borel set $E \subset \mathbb{R}^{n-1}, \nu(E)^{\frac{n-1}{n p}} \leq c_{n, p} \mu(E)^{\frac{1}{p}}$. In particular, $\nu$ is absolutely continuous with respect to $\mu$. By Radon-Nikydom theorem (EG, section 1.6]) we have

$$
\nu(E)=\int_{E} g d \mu
$$


Moreover for $\mu$ a.e. $\xi \in \mathbb{R}^{n-1}$

$$
g(\xi)=\lim _{r \rightarrow 0^{+}} \frac{\nu\left(\bar{B}_{r}^{n-1}(\xi)\right)}{\mu\left(\bar{B}_{r}^{n-1}(\xi)\right)} .
$$

Let $J=\left\{\xi \in \mathbb{R}^{n-1}: \mu(\{\xi\})>0\right\}$, then $J$ is countable. Moreover, for $\xi \notin J$, we have

$$
g(\xi) \leq \lim \inf _{r \rightarrow 0^{+}} c_{n, p}^{\frac{n p}{n-1}} \mu\left(\bar{B}_{r}^{n-1}(\xi)\right)^{\frac{1}{n-1}}=0 .
$$

Hence $\nu=|P f|^{\frac{n p}{n-1}} d x+\sum_{j} \nu_{j} \delta_{\zeta_{j}}$. For the third assertion, if we know $\nu\left(\mathbb{R}^{n-1}\right)^{\frac{n-1}{n p}} \geq$ $c_{n, p} \mu\left(\mathbb{R}^{n-1}\right)^{\frac{1}{p}}$, then

$$
\left(\sum_{j} \nu_{j}\right)^{\frac{n-1}{n p}} \geq c_{n, p}\left(\sum_{j} \mu_{j}\right)^{1 / p} \geq\left(\sum_{j} \nu_{j}^{\frac{n-1}{n}}\right)^{1 / p}
$$

hence

$$
\left(\sum_{j} \nu_{j}\right)^{\frac{n-1}{n}} \geq \sum_{j} \nu_{j}^{\frac{n-1}{n}}
$$

Since $0<\frac{n-1}{n}<1$, we see at most one $\nu_{j}$ is nonzero.

Now we are ready to prove Theorem 3.1 .

Proof of Theorem 3.1. For $r>0$, let

$$
\phi_{i}(r)=\sup _{\zeta \in \mathbb{R}^{n-1}} \int_{B_{r}^{n-1}(\zeta)}\left|f_{i}\right|^{p} d \xi .
$$

Then $\phi_{i}:(0, \infty) \rightarrow[0,1]$ is a continuous nondecreasing function with

$$
\lim _{r \rightarrow 0^{+}} \phi_{i}(r)=0, \quad \lim _{r \rightarrow \infty} \phi_{i}(r)=1 .
$$

By introducing dilation factor $\lambda_{i}$ and translation by $\zeta_{i}$, we may assume

$$
\phi_{i}(1)=1 / 2=\int_{B_{1}}\left|f_{i}\right|^{p} d \xi
$$

After passing to a subsequence, we may find $f \in L^{p}\left(\mathbb{R}^{n-1}\right)$ such that

$$
f_{i} \rightarrow f \text { in } L^{p}\left(\mathbb{R}^{n-1}\right),\left|f_{i}\right|^{p} d \xi \rightarrow \mu \text { in } \mathcal{M}\left(\mathbb{R}^{n-1}\right),\left|P f_{i}\right|^{\frac{n p}{n-1}} d x \rightarrow \nu \text { in } \mathcal{M}\left(\overline{\mathbb{R}_{+}^{n}}\right) .
$$

In particular, this implies $\mu\left(\bar{B}_{1}^{n-1}\right) \geq 1 / 2$. We claim $\mu\left(\mathbb{R}^{n-1}\right)=1$. If not, then $\mu\left(\mathbb{R}^{n-1}\right)=\theta \in(0,1)$. For $\varepsilon>0$ small, we claim that after passing to a subsequence, we may find $r_{0}>0$ and a sequence $r_{i} \rightarrow \infty$ such that

$$
\theta-\varepsilon<\int_{B_{r_{0}}^{n-1}}\left|f_{i}\right|^{p} d \xi \leq \int_{B_{r_{0}+2 r_{i}}^{n-1}}\left|f_{i}\right|^{p} d \xi<\theta+\varepsilon .
$$

Indeed, fix $r_{0}>0$ such that $\mu\left(B_{r_{0}}^{n-1}\right)>\theta-\varepsilon$, then for $i$ large enough, we have $\int_{B_{r_{0}}^{n-1}}\left|f_{i}\right|^{p} d \xi>\theta-\varepsilon$. On the other hand, since $\mu\left(\bar{B}_{r_{0}+2 i}^{n-1}\right) \leq \theta<\theta+\varepsilon$, we may 
inductively define $n_{i}>i, n_{i+1}>n_{i}$ such that $\int_{B_{r_{0+2 i}}^{n-1}}\left|f_{n_{i}}\right|^{p} d x<\theta+\varepsilon$. Replacing $f_{i}$ by $f_{n_{i}}$ we get the needed claim. Let

$$
g_{i}=f_{i} \chi_{B_{r_{0}}^{n-1}}, \quad h_{i}=f_{i} \chi_{\mathbb{R}^{n-1} \backslash B_{r_{0}+2 r_{i}}^{n-1}} .
$$

Since

we see

$$
\int_{B_{r_{0}+2 r_{i}}^{n-1} \backslash B_{r_{0}}^{n-1}}\left|f_{i}\right|^{p} d \xi \leq 2 \varepsilon
$$

$$
\left|f_{i}-g_{i}-h_{i}\right|_{L^{p}\left(\mathbb{R}^{n-1}\right)} \leq c(n, p) \varepsilon^{1 / p}
$$

Note that

$$
\begin{aligned}
& || P g_{i}+\left.P h_{i}\right|^{\frac{n p}{n-1}}-\left|P g_{i}\right|^{\frac{n p}{n-1}}-\left|P h_{i}\right|^{\frac{n p}{n-1}} \mid \\
& \leq c(n, p)\left(\left|P g_{i}\right|^{\frac{n p}{n-1}-1}\left|P h_{i}\right|+\left|P g_{i}\right|\left|P h_{i}\right|^{\frac{n p}{n-1}-1}\right) .
\end{aligned}
$$

On the other hand,

$$
\begin{aligned}
& \int_{\mathbb{R}_{+}^{n}}\left|P g_{i}\right|^{\frac{n p}{n-1}-1}\left|P h_{i}\right| d x
\end{aligned}
$$

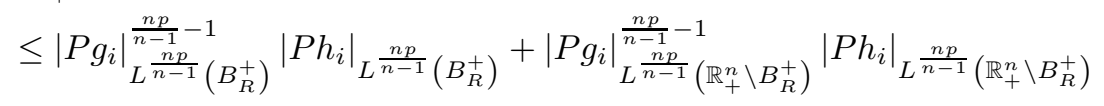

$$
\begin{aligned}
& \leq c(n, p) R^{\frac{n-1}{p}} r_{i}^{-\frac{n-1}{p}}+c(n, p) r_{0}^{\frac{(p-1)(n p-n+1)}{p}}\left|\frac{x_{n}}{\left[\left(\left(\left|x^{\prime}\right|-r_{0}\right)^{+}\right)^{2}+x_{n}^{2}\right]^{n / 2}}\right|_{L^{\frac{n p}{n-1}}\left(\mathbb{R}_{+}^{n} \backslash B_{R}^{+}\right)}^{\frac{n p}{n-1}-1}
\end{aligned}
$$

this implies

$$
\begin{aligned}
& \limsup _{i \rightarrow \infty} \int_{\mathbb{R}_{+}^{n}}\left|P g_{i}\right|^{\frac{n p}{n-1}-1}\left|P h_{i}\right| d x \\
& \leq c(n, p) r_{0}^{\frac{(p-1)(n p-n+1)}{p}}\left|\frac{x_{n}}{\left[\left(\left(\left|x^{\prime}\right|-r_{0}\right)^{+}\right)^{2}+x_{n}^{2}\right]^{n / 2}}\right|_{L^{\frac{n p}{n-1}}\left(\mathbb{R}_{+}^{n} \backslash B_{R}^{+}\right)}^{\frac{n p}{n-1}-1} .
\end{aligned}
$$

Let $R \rightarrow \infty$, we see

$$
\lim _{i \rightarrow \infty} \int_{\mathbb{R}_{+}^{n}}\left|P g_{i}\right|^{\frac{n p}{n-1}-1}\left|P h_{i}\right| d x=0
$$

Similarly,

$$
\lim _{i \rightarrow \infty} \int_{\mathbb{R}_{+}^{n}}\left|P g_{i}\right|\left|P h_{i}\right|^{\frac{n p}{n-1}-1} d x=0
$$

Hence

$$
\lim _{i \rightarrow \infty} \int_{\mathbb{R}_{+}^{n}}\left|P g_{i}+P h_{i}\right|^{\frac{n p}{n-1}}-\left|P g_{i}\right|^{\frac{n p}{n-1}}-\left|P h_{i}\right|^{\frac{n p}{n-1}} \mid d x=0 .
$$


Since

$$
\begin{aligned}
& \int_{\mathbb{R}_{+}^{n}}\left|P g_{i}\right|^{\frac{n p}{n-1}} d x \leq c_{n, p}^{\frac{n p}{n-1}}\left|g_{i}\right|_{L^{p}\left(\mathbb{R}^{n-1}\right)}^{\frac{n p}{n-1}} \leq c_{n, p}^{\frac{n p}{n-1}}(\theta+\varepsilon)^{\frac{n}{n-1}} \\
& \int_{\mathbb{R}_{+}^{n}}\left|P h_{i}\right|^{\frac{n p}{n-1}} d x \leq c_{n, p}^{\frac{n p}{n-1}}\left|h_{i}\right|_{L^{p}\left(\mathbb{R}^{n-1}\right)}^{\frac{n p}{n-1}} \leq c_{n, p}^{\frac{n p}{n-1}}(1-\theta+\varepsilon)^{\frac{n}{n-1}}
\end{aligned}
$$

we see

$$
\begin{aligned}
& c_{n, p}^{\frac{n p}{n-1}}+o(1) \\
& =\int_{\mathbb{R}_{+}^{n}}\left|P f_{i}\right|^{\frac{n p}{n-1}} d x \\
& \leq\left(\left|P g_{i}+P h_{i}\right|_{L^{\frac{n p}{n-1}}\left(\mathbb{R}_{+}^{n}\right)}+c(n, p) \varepsilon^{1 / p}\right)^{\frac{n p}{n-1}} \\
& \leq \int_{\mathbb{R}_{+}^{n}}\left|P g_{i}+P h_{i}\right|^{\frac{n p}{n-1}} d x+c(n, p) \varepsilon^{1 / p} \\
& \leq \int_{\mathbb{R}_{+}^{n}}\left(\left|P g_{i}\right|^{\frac{n p}{n-1}}+\left|P h_{i}\right|^{\frac{n p}{n-1}}\right) d x+c(n, p) \varepsilon^{1 / p}+o(1) \\
& \leq c_{n, p}^{\frac{n p}{n-1}}(\theta+\varepsilon)^{\frac{n}{n-1}}+c_{n, p}^{\frac{n p}{n-1}}(1-\theta+\varepsilon)^{\frac{n}{n-1}}+c(n, p) \varepsilon^{1 / p}+o(1) .
\end{aligned}
$$

Letting $i \rightarrow \infty$ and then $\varepsilon \rightarrow 0^{+}$, we see

$$
1 \leq \theta^{\frac{n}{n-1}}+(1-\theta)^{\frac{n}{n-1}}
$$

This gives us a contradiction since $\frac{n}{n-1}>1$. Hence $\mu\left(\mathbb{R}^{n-1}\right)=1$. Next we claim $\nu\left(\overline{\mathbb{R}_{+}^{n}}\right)=c_{n, p}^{\frac{n p}{n-1}}$. Indeed, for any $\varepsilon>0$ small, we may find $r>0$ such that $\mu\left(B_{r}^{n-1}\right)>1-\varepsilon$, this implies $\int_{B_{r}^{n-1}}\left|f_{i}\right|^{p} d \xi>1-\varepsilon$ when $i$ is large enough. Hence $\int_{\mathbb{R}^{n-1} \backslash B_{r}^{n-1}}\left|f_{i}\right|^{p} d \xi \leq \varepsilon$. Let $g_{i}=f_{i} \chi_{B_{r}^{n-1}}$ and $h_{i}=f_{i} \chi_{\mathbb{R}^{n-1} \backslash B_{r}^{n-1}}$, then

$$
\begin{aligned}
&\left|P h_{i}\right|_{L^{\frac{n p}{n-1}}\left(\mathbb{R}_{+}^{n}\right)} \leq c(n, p) \varepsilon^{1 / p}, \\
&\left|\left(P g_{i}\right)(x)\right| \leq c(n, p) r^{\frac{(n-1)(p-1)}{p}} \frac{x_{n}}{\left[\left(\left(\left|x^{\prime}\right|-r\right)^{+}\right)^{2}+x_{n}^{2}\right]^{n / 2}} .
\end{aligned}
$$

This implies

$$
\begin{aligned}
& \int_{\mathbb{R}^{n} \backslash B_{R}^{+}}\left|P f_{i}\right|^{\frac{n p}{n-1}} d x \\
& \leq c(n, p) \varepsilon^{\frac{n}{n-1}}+c(n, p) r^{n(p-1)}\left|\frac{x_{n}}{\left[\left(\left(\left|x^{\prime}\right|-r\right)^{+}\right)^{2}+x_{n}^{2}\right]^{n / 2}}\right|_{L^{\frac{n p}{n-1}}\left(\mathbb{R}^{n} \backslash B_{R}^{+}\right)}^{\frac{n p}{n-1}}
\end{aligned}
$$


Taking a limit for $i \rightarrow \infty$, we see

$\nu\left(\overline{\mathbb{R}_{+}^{n}}\right) \geq \nu\left(\overline{B_{R}^{+}}\right)$

$$
\geq c_{n, p}^{\frac{n p}{n-1}}-c(n, p) \varepsilon^{\frac{n}{n-1}}-c(n, p) r^{n(p-1)}\left|\frac{x_{n}}{\left[\left(\left(\left|x^{\prime}\right|-r\right)^{+}\right)^{2}+x_{n}^{2}\right]^{n / 2}}\right|_{L^{\frac{n p}{n-1}}\left(\mathbb{R}^{n} \backslash B_{R}^{+}\right)}^{\frac{n p}{n-1}} .
$$

let $R \rightarrow \infty$ then $\varepsilon \rightarrow 0^{+}$, we see $\nu\left(\overline{\mathbb{R}_{+}^{n}}\right)=c_{n, p}^{\frac{n p}{n-1}}$.

By Proposition 3.1 we know there exists a countable set of points $\zeta_{j} \in \mathbb{R}^{n-1}$ such that

$$
\nu=|P f|^{\frac{n p}{n-1}} d x+\sum_{j} \nu_{j} \delta_{\zeta_{j}}, \quad \mu \geq|f|^{p} d \xi+\sum_{j} \mu_{j} \delta_{\zeta_{j}},
$$

here $\mu_{j}=\mu\left(\left\{\zeta_{j}\right\}\right)$ and

$$
\nu_{j}^{\frac{n-1}{n p}} \leq c_{n, p} \mu_{j}^{\frac{1}{p}} .
$$

If $f=0$, then $\nu\left(\mathbb{R}^{n-1}\right)=c_{n, p}^{\frac{n p}{n-1}}$ and hence $\nu\left(\mathbb{R}^{n-1}\right)^{\frac{n-1}{n p}}=c_{n, p} \mu\left(\mathbb{R}^{n-1}\right)^{\frac{1}{p}}$. This implies for some $\zeta_{1} \in \mathbb{R}^{n-1}, \nu=c_{n, p}^{\frac{n p}{n-1}} \delta_{\zeta_{1}}$. In particular, $\mu\left(\left\{\zeta_{1}\right\}\right) \geq 1$ and this implies $\mu=\delta_{\zeta_{1}}$. But

$$
\int_{B_{1}^{n-1}\left(\zeta_{1}\right)}\left|f_{i}\right|^{p} d \xi \leq 1 / 2
$$

implies $\mu\left(B_{1}^{n-1}\left(\zeta_{1}\right)\right) \leq 1 / 2$. This gives us a contradiction. Hence $f \neq 0$. Now

$$
c_{n, p}^{\frac{n p}{n-1}}=|P f|_{L^{\frac{n p}{n-1}}\left(\mathbb{R}_{+}^{n}\right)}^{\frac{n p}{n-1}}+\sum_{j} \nu_{j} \leq c_{n, p}^{\frac{n p}{n-1}}|f|_{L^{p}\left(\mathbb{R}^{n-1}\right)}^{\frac{n p}{n-1}}+c_{n, p}^{\frac{n p}{n-1}} \sum_{j} \mu_{j}^{\frac{n}{n-1}},
$$

hence

But since

$$
1 \leq|f|_{L^{p}\left(\mathbb{R}^{n-1}\right)}^{\frac{n p}{n-1}}+\sum_{j} \mu_{j}^{\frac{n}{n-1}}
$$

$$
1 \geq|f|_{L^{p}\left(\mathbb{R}^{n-1}\right)}^{p}+\sum_{j} \mu_{j}
$$

and $\frac{n}{n-1}>1$, we see $\mu_{j}=0$ and $|f|_{L^{p}\left(\mathbb{R}^{n-1}\right)}=1$. This implies $f_{i} \rightarrow f$ in $L^{p}\left(\mathbb{R}^{n-1}\right)$.

\section{The EXistence of maXimizing Functions for SharP INEQUALities By SYMMETRIZATION}

Following Lieb ([Li2 $)$, using the method of symmetrization we will show all the maximizers of variational problem (3.1) are radial symmetric with respect to some point and we will give another approach to the existence of maximizing functions.

Let $u$ be a measurable function on $\mathbb{R}^{n}$, the symmetric rearrangement of $u$ is the nonnegative lower semi-continuous radial decreasing function $u^{*}$ which has the same distribution as $u$. It satisfies the following important Riesz rearrangement inequality ([LiL, p87]): for any nonnegative measurable functions $u, v, w$ on $\mathbb{R}^{n}$, we have

$$
\int_{\mathbb{R}^{n}} d x \int_{\mathbb{R}^{n}} u(x) v(y-x) w(y) d y \leq \int_{\mathbb{R}^{n}} d x \int_{\mathbb{R}^{n}} u^{*}(x) v^{*}(y-x) w^{*}(y) d y .
$$


Using the fact $|w|_{L^{p}\left(\mathbb{R}^{n}\right)}=\left|w^{*}\right|_{L^{p}\left(\mathbb{R}^{n}\right)}$ for $p>0$, we see for $1 \leq p \leq \infty$,

$$
|u * v|_{L^{p}\left(\mathbb{R}^{n}\right)} \leq\left|u^{*} * v^{*}\right|_{L^{p}\left(\mathbb{R}^{n}\right)} .
$$

Moreover if $u$ is nonnegative radial symmetric and strictly decreasing in the radial direction, $v$ is nonnegative, $1<p<\infty$ and

$$
|u * v|_{L^{p}\left(\mathbb{R}^{n}\right)}=\left|u * v^{*}\right|_{L^{p}\left(\mathbb{R}^{n}\right)}<\infty,
$$

then for some $x_{0} \in \mathbb{R}^{n}$, we have $v(x)=v^{*}\left(x-x_{0}\right)$.

Indeed, we may assume $v$ is not identically zero. Choose a nonnegative $w \in$ $L^{p^{\prime}}\left(\mathbb{R}^{n}\right)$ with $|w|_{L^{p^{\prime}\left(\mathbb{R}^{n}\right)}}=1$ such that

$$
|u * v|_{L^{p}\left(\mathbb{R}^{n}\right)}=\int_{\mathbb{R}^{n}}(u * v)(y) w(y) d y .
$$

Then we have

$$
\begin{aligned}
|u * v|_{L^{p}\left(\mathbb{R}^{n}\right)} & =\int_{\mathbb{R}^{n}} d x \int_{\mathbb{R}^{n}} u(x) v(y-x) w(y) d y \\
& \leq \int_{\mathbb{R}^{n}} d x \int_{\mathbb{R}^{n}} u(x) v^{*}(y-x) w^{*}(y) d y \\
& =\int_{\mathbb{R}^{n}}\left(u * v^{*}\right)(y) w^{*}(y) d y \\
& \leq\left|u * v^{*}\right|_{L^{p}\left(\mathbb{R}^{n}\right)}=|u * v|_{L^{p}\left(\mathbb{R}^{n}\right)},
\end{aligned}
$$

hence all the inequalities become equalities. It follows from the Lieb's strong version of Riesz rearrangement inequality ([i1]) that for some $x_{0} \in \mathbb{R}^{n}, v(x)=v^{*}\left(x-x_{0}\right)$.

Theorem 4.1. Assume $n \geq 2$ and $1<p<\infty$, then the value

$$
c_{n, p}^{\frac{n p}{n-1}}=\sup \left\{\int_{\mathbb{R}_{+}^{n}}|P f|^{\frac{n p}{n-1}} d x: f \in L^{p}\left(\mathbb{R}^{n-1}\right),|f|_{L^{p}\left(\mathbb{R}^{n-1}\right)}=1\right\},
$$

is attained by some functions. After multiplying by a nonzero constant, every maximizer $f$ is nonnegative, radial symmetric with respect to some point, strictly decreasing in the radial direction and it satisfies

$$
f(\xi)^{p-1}=\int_{\mathbb{R}_{+}^{n}} P(x, \xi)(P f)(x)^{\frac{n p}{n-1}-1} d x .
$$

If $n \geq 3$ and $p=\frac{2(n-1)}{n-2}$, then any maximizer must be of the form

$$
f(\xi)= \pm c(n)\left(\frac{\lambda}{\lambda^{2}+\left|\xi-\xi_{0}\right|^{2}}\right)^{\frac{n-2}{2}}
$$

for some $\lambda>0, \xi_{0} \in \mathbb{R}^{n-1}$. In particular, $c_{n, \frac{2(n-1)}{n-2}}=n^{-\frac{n-2}{2(n-1)} \omega_{n}^{-\frac{n-2}{2 n(n-1)}}}$.

If $n \geq 3$ and $p=\frac{2(n-1)}{n}$, then any maximizer must be of the form

$$
f(\xi)= \pm c(n)\left(\frac{\lambda}{\lambda^{2}+\left|\xi-\xi_{0}\right|^{2}}\right)^{\frac{n}{2}}
$$

for some $\lambda>0, \xi_{0} \in \mathbb{R}^{n-1}$. In particular, $c_{n, \frac{2(n-1)}{n}}=\frac{1}{\sqrt{2(n-2) \sqrt[4]{\pi}}}\left(\frac{(n-2) !}{\Gamma\left(\frac{n-1}{2}\right)}\right)^{\frac{1}{2(n-1)}}$. 
Proof. Assume $f_{i}$ is a maximizing sequence. Since $\left|f_{i}^{*}\right|_{L^{p}\left(\mathbb{R}^{n-1}\right)}=\left|f_{i}\right|_{L^{p}\left(\mathbb{R}^{n-1}\right)}=1$ and

$$
\begin{aligned}
\left|P f_{i}\right|_{L^{\frac{n p}{n-1}}}^{\frac{n p}{n-1}}\left(\mathbb{R}_{+}^{n}\right) & =\int_{0}^{\infty}\left|P_{x_{n}} * f_{i}\right|_{L^{\frac{n p}{n-1}}\left(\mathbb{R}^{n-1}\right)}^{\frac{n p}{n-1}} d x_{n} \\
& \leq \int_{0}^{\infty}\left|P_{x_{n}} * f_{i}^{*}\right|_{L^{\frac{n n p}{n-1}}\left(\mathbb{R}^{n-1}\right)}^{\frac{n p}{n-1}} d x_{n}=\left|P f_{i}^{*}\right|_{L^{\frac{n p}{n-1}}}^{\frac{n p}{n-1}\left(\mathbb{R}_{+}^{n}\right)}
\end{aligned}
$$

we see $f_{i}^{*}$ is again a maximizing sequence. Hence we may assume $f_{i}$ is a nonnegative radial decreasing function.

For any $f \in L^{p}\left(\mathbb{R}^{n-1}\right)$ and any $\lambda>0$, we let $f^{\lambda}(\xi)=\lambda^{-\frac{n-1}{p}} f\left(\frac{\xi}{\lambda}\right)$, then it is clear that $\left|f^{\lambda}\right|_{L^{p}\left(\mathbb{R}^{n-1}\right)}=|f|_{L^{p}\left(\mathbb{R}^{n-1}\right)}$ and $\left|P f^{\lambda}\right|_{L^{\frac{n p}{n-1}\left(\mathbb{R}_{+}^{n}\right)}}=|P f|_{L^{n-1}\left(\mathbb{R}_{+}^{n}\right)}$. For convenience, denote $e_{1}=(1,0, \cdots, 0) \in \mathbb{R}^{n}$ and

$$
a_{i}=\sup _{\lambda>0} f_{i}^{\lambda}\left(e_{1}^{\prime}\right)=\sup _{\lambda>0} \lambda^{-\frac{n-1}{p}} f_{i}\left(\frac{e_{1}^{\prime}}{\lambda}\right) \text {. }
$$

It follows that

$$
0 \leq f_{i}(\xi) \leq a_{i}|\xi|^{-\frac{n-1}{p}}
$$

and hence

$$
\left|f_{i}\right|_{L^{p, \infty}\left(\mathbb{R}^{n-1}\right)} \leq \omega_{n-1}^{1 / p} a_{i}
$$

Now

$$
\begin{aligned}
\left|P f_{i}\right|_{L^{\frac{n p}{n-1}}\left(\mathbb{R}_{+}^{n}\right)} & \leq c(n, p)\left|f_{i}\right|_{L^{p, \frac{n p}{n-1}}\left(\mathbb{R}_{+}^{n}\right)} \\
& \leq c(n, p)\left|f_{i}\right|_{L^{p}\left(\mathbb{R}_{+}^{n}\right)}^{\frac{n-1}{n}}\left|f_{i}\right|_{L^{p, \infty}\left(\mathbb{R}_{+}^{n}\right)}^{\frac{1}{n}} \\
& \leq c(n, p) a_{i}^{1 / n},
\end{aligned}
$$

this implies $a_{i} \geq c(n, p)>0$. We may choose $\lambda_{i}>0$ such that $f_{i}^{\lambda_{i}}\left(e_{1}^{\prime}\right) \geq c(n, p)>$ 0 . Replacing $f_{i}$ by $f_{i}^{\lambda_{i}}$ we may assume $f\left(e_{1}^{\prime}\right) \geq c(n, p)>0$. On the other hand, since $f_{i}$ is nonnegative radial decreasing and $\left|f_{i}\right|_{L^{p}\left(\mathbb{R}^{n-1}\right)}=1$, we see

$$
\left|f_{i}(\xi)\right| \leq \omega_{n-1}^{-1 / p}|\xi|^{-(n-1) / p}
$$

Hence after passing to a subsequence, we may find a nonnegative radial decreasing function $f$ such that $f_{i} \rightarrow f$ a.e.. It follows that $f(\xi) \geq c(n, p)>0$ for $|\xi| \leq 1$, $f_{i} \rightarrow f$ in $L^{p}\left(\mathbb{R}^{n-1}\right)$ and $|f|_{L^{p}\left(\mathbb{R}^{n-1}\right)} \leq 1$. Since

$$
\left.\int_{\mathbb{R}^{n-1}}|| f_{i}\right|^{p}-|f|^{p}-\left|f_{i}-f\right|^{p} \mid d \xi \rightarrow 0
$$

we see

$$
\begin{aligned}
\left|f_{i}-f\right|_{L^{p}\left(\mathbb{R}^{n-1}\right)}^{p} & =\left|f_{i}\right|_{L^{p}\left(\mathbb{R}^{n-1}\right)}^{p}-|f|_{L^{p}\left(\mathbb{R}^{n-1}\right)}^{p}+o(1) \\
& =1-|f|_{L^{p}\left(\mathbb{R}^{n-1}\right)}^{p}+o(1) .
\end{aligned}
$$

On the other hand, since $\left(P f_{i}\right)(x) \rightarrow(P f)(x)$ for $x \in \mathbb{R}_{+}^{n}$ and $\left|P f_{i}\right|_{L^{\frac{n p}{n-1}}\left(\mathbb{R}_{+}^{n}\right)} \leq$ $c_{n, p}$, we see

$$
\begin{aligned}
\left|P f_{i}\right|_{L^{\frac{n p}{n-1}}\left(\mathbb{R}_{+}^{n}\right)}^{\frac{n p}{n-1}} & =|P f|_{L^{\frac{n p}{n-1}}\left(\mathbb{R}_{+}^{n}\right)}^{\frac{n p}{n-1}}+\left|P f_{i}-P f\right|_{L^{\frac{n p}{n-1}}\left(\mathbb{R}_{+}^{n}\right)}^{\frac{n p}{n-1}}+o(1) \\
& \leq c_{n, p}^{\frac{n p}{n-1}}|f|_{L^{p}\left(\mathbb{R}^{n-1}\right)}^{\frac{n p}{n-1}}+c_{n, p}^{\frac{n p}{n-1}}\left|f_{i}-f\right|_{L^{p}\left(\mathbb{R}^{n-1}\right)}^{\frac{n p}{n-1}}+o(1) .
\end{aligned}
$$


Hence

$$
1 \leq|f|_{L^{p}\left(\mathbb{R}^{n-1}\right)}^{\frac{n p}{n-1}}+\left|f_{i}-f\right|_{L^{p}\left(\mathbb{R}^{n-1}\right)}^{\frac{n p}{n-1}}+o(1) .
$$

Let $i \rightarrow \infty$, we see

$$
1 \leq|f|_{L^{p}\left(\mathbb{R}^{n-1}\right)}^{\frac{n p}{n-1}}+\left(1-|f|_{L^{p}\left(\mathbb{R}^{n-1}\right)}^{p}\right)^{\frac{n}{n-1}} .
$$

Since $\frac{n}{n-1}>1$ and $f \neq 0$, we see $|f|_{L^{p}\left(\mathbb{R}^{n-1}\right)}=1$. Hence $f_{i} \rightarrow f$ in $L^{p}\left(\mathbb{R}^{n-1}\right)$ and $f$ is a maximizer. This implies the existence of an extremal function.

Assume $f \in L^{p}\left(\mathbb{R}^{n-1}\right)$ is a maximizer, then so is $|f|$. Hence $|P f|_{L^{\frac{n p}{n-1}}\left(\mathbb{R}_{+}^{n}\right)}=$ $|P| f||_{L^{\frac{n p}{n-1}}\left(\mathbb{R}_{+}^{n}\right)}$. On the other hand, since $|(P f)(x)| \leq P(|f|)(x)$ for $x \in \mathbb{R}_{+}^{n}$, we see $|P f|=P(|f|)$ and this implies either $f \geq 0$ or $f \leq 0$. Assume $f \geq 0$, then the Euler-Lagrange equation is given by

$$
\int_{\mathbb{R}_{+}^{n}} P(x, \xi)(P f)(x)^{\frac{n p}{n-1}-1} d x=c \cdot f(\xi)^{p-1} .
$$

Here $c$ is a constant. Using the fact $|f|_{L^{p}\left(\mathbf{R}^{n-1}\right)}=1$, we see

$$
c=|P f|_{L^{\frac{n p}{n-1}}\left(\mathbb{R}_{+}^{n}\right)}^{\frac{n p}{n-1}}=c_{n, p}^{\frac{n p}{n-1}} .
$$

After scaling by a positive constant we get

$$
\int_{\mathbb{R}_{+}^{n}} P(x, \xi)(P f)(x)^{\frac{n p}{n-1}-1} d x=f(\xi)^{p-1} .
$$

On the other hand, we know for $x_{n}>0,\left|P_{x_{n}} * f\right|_{L^{\frac{n p}{n-1}}\left(\mathbb{R}^{n-1}\right)}=\left|P_{x_{n}} * f^{*}\right|_{L^{\frac{n p}{n-1}}\left(\mathbb{R}^{n-1}\right)}$, this implies $f(\xi)=f^{*}\left(\xi-\xi_{0}\right)$ for some $\xi_{0}$. It follows from the Euler-Lagrange equation that $f$ must be strictly decreasing along the radial direction.

For the case when $p=\frac{2(n-1)}{n-2}$, we first observe that if $f \in L^{\frac{2(n-1)}{n-2}}\left(\mathbb{R}^{n-1}\right)$, let $u=P f, \tilde{f}(\xi)=\frac{1}{|\xi|^{n-2}} f\left(\frac{\xi}{|\xi|^{2}}\right)$ and $\widetilde{u}(x)=\frac{1}{|x|^{n-2}} u\left(\frac{x}{|x|^{2}}\right)$, then we have $\widetilde{u}=P \widetilde{f}$, $|\tilde{f}|_{L^{\frac{2(n-1)}{n-2}}\left(\mathbb{R}^{n-1}\right)}=|f|_{L^{\frac{2(n-1)}{n-2}}\left(\mathbb{R}^{n-1}\right)}$ and $|\widetilde{u}|_{L^{\frac{2 n}{n-2}}\left(\mathbb{R}_{+}^{n}\right)}=|u|_{L^{\frac{2 n}{n-2}}\left(\mathbb{R}_{+}^{n}\right)}$. This is the conformal invariance property for the particular power. As a consequence, if $f$ is a maximizer which is nonnegative and radial, then $\frac{1}{|\xi|^{n-2}} f\left(\frac{\xi}{|\xi|^{2}}-e_{1}^{\prime}\right)$ is a maximizer too. In particular, $\frac{1}{|\xi|^{n-2}} f\left(\frac{\xi}{|\xi|^{2}}-e_{1}^{\prime}\right)$ is radial with respect to some point. To find such $f$, we prove the following facts.

Proposition 4.1. Let $n \geq 2$, $u$ be a function on $\mathbb{R}^{n}$ which is radial with respect to the origin, $0<u(x)<\infty$ for $x \neq 0, e_{1}=(1,0, \cdots, 0), \alpha \in \mathbb{R}, \alpha \neq 0$. If $v(x)=|x|^{\alpha} u\left(\frac{x}{|x|^{2}}-e_{1}\right)$ is radial with respect to some point, then either $u(x)=$ $\left(c_{1}|x|^{2}+c_{2}\right)^{\alpha / 2}$ for some $c_{1} \geq 0, c_{2}>0$ or

$$
u(x)=\left\{\begin{array}{l}
c_{1}|x|^{\alpha}, \text { if } x \neq 0, \\
c_{2}, \text { if } x=0,
\end{array}\right.
$$

for some $c_{1}>0$ and $c_{2}$, an arbitrary number. 
Proof. First we observe that $\left|\frac{x}{|x|^{2}}-e_{1}\right|=1$ if and only if $x_{1}=\frac{1}{2}$. For $r>0, r \neq 1$, we have $\left|\frac{x}{|x|^{2}}-e_{1}\right|=r$ if and only if $x \in \partial B \frac{r}{\left|r^{2}-1\right|}\left(\frac{e_{1}}{1-r^{2}}\right)$. By scaling, we may assume $u\left(e_{1}\right)=1$. Then $v\left(\frac{1}{2}, x^{\prime \prime}\right)=\left(\frac{1}{4}+\left|x^{\prime \prime}\right|^{2}\right)^{\alpha / 2}$. Assume $v$ is symmetric with respect to $z=\left(z_{1}, z^{\prime \prime}\right)$. Then $v\left(\frac{1}{2}, \cdot\right)$ is symmetric with respect to $z^{\prime \prime}$, hence $z^{\prime \prime}=0$. Denote $z=a e_{1}$, we claim $0 \leq a \leq 1$. If this is not the case, then we may find a $r>0, r \neq 1$ such that $a=\frac{1}{1-r^{2}}$. Now on $\partial B \frac{r}{\left|r^{2}-1\right|}\left(\frac{e_{1}}{1-r^{2}}\right), v(x)=|x|^{\alpha} u\left(r e_{1}\right)$ and it is not a constant function, contradiction. For $x=\left(\frac{1}{2}, x^{\prime \prime}\right)$, we have

$$
v(x)=\left(\left|x-a e_{1}\right|^{2}+a-a^{2}\right)^{\alpha / 2} .
$$

Hence

$$
v(x)=\left(\left|x-a e_{1}\right|^{2}+a-a^{2}\right)^{\alpha / 2}=\left(|x|^{2}-2 a x_{1}+a\right)^{\alpha / 2}
$$

for $\left|x-a e_{1}\right| \geq\left|\frac{1}{2}-a\right|$. When $a=\frac{1}{2}$, we see $v(x)=\left(|x|^{2}-2 a x_{1}+a\right)^{\alpha / 2}$ for all $x$. This implies $u(x)=\left(\frac{1}{2}|x|^{2}+\frac{1}{2}\right)^{\alpha / 2}$. Hence we assume $a \neq \frac{1}{2}$ from now on. Without losing of generality, we assume $0 \leq a<\frac{1}{2}$. We claim that

$$
v(x)=\left(|x|^{2}-2 a x_{1}+a\right)^{\alpha / 2}
$$

for all $x \neq 0$. To see this, we first make the following observation. Assume for some given $r>0, r \neq 1$ and for some $y \in \partial B \frac{r}{\left|r^{2}-1\right|}\left(\frac{e_{1}}{1-r^{2}}\right)$, (4.2) is true for $y$, then it is true for all $x \in \partial B \frac{r}{\left|r^{2}-1\right|}\left(\frac{e_{1}}{1-r^{2}}\right)$. Indeed, for $x$ on such a sphere, we have

$$
\frac{1-2 x_{1}}{|x|^{2}}=r^{2}-1
$$

Hence

$$
\begin{aligned}
v(x) & =|x|^{\alpha} u\left(r e_{1}\right)=|x|^{\alpha}|y|^{-\alpha}\left(|y|^{2}-2 a y_{1}+a\right)^{\alpha / 2} \\
& =|x|^{\alpha}\left(1+\frac{a\left(1-2 x_{1}\right)}{|x|^{2}}\right)^{\alpha / 2}=\left(|x|^{2}-2 a x_{1}+a\right)^{\alpha / 2} .
\end{aligned}
$$

Note that we know (4.2) is true for $x=t e_{1}$ with $t \in\left(-\infty,-\frac{1}{2}\right]$. By the above observation we know it is also true for $t e_{1}$ with $t \in\left[\frac{1}{4}, \frac{1}{2}\right]$. This implies it is true for $t e_{1}$ with $t \in\left(-\infty,-\frac{1}{4}\right]$. Go back we see it is true for $t e_{1}$ with $t \in\left[\frac{1}{6}, \frac{1}{2}\right]$. Keep this procedure going, we see (4.2) is true for all $t e_{1}$ with $t \neq 0$. Hence it is true for all $x \neq 0$. This implies $u(x)=\left(a|x|^{2}+1-a\right)^{\alpha / 2}$.

Remark 4.1. The case when $\alpha=0$ is a little bit different. However one has: Let $n \geq 2$, $u$ be a function on $\mathbb{R}^{n}$ which is radial with respect to the origin, $e_{1}=$ $(1,0, \cdots, 0)$. If $v(x)=u\left(\frac{x}{|x|^{2}}-e_{1}\right)$ is radial with respect to some point, then either

$$
u(x)=\left\{\begin{array}{l}
c_{1}, \text { if } x=0 \\
c_{2}, \text { if } x \neq 0
\end{array}\right.
$$


or there exists $r>0, r \neq 1$ such that

$$
u(x)= \begin{cases}c_{1}, & \text { if }|x|<r \\ c_{2}, & \text { if }|x|=r \\ c_{3}, & \text { if }|x|>r .\end{cases}
$$

Here $c_{i}$ 's are arbitrary constants.

Proof of Theorem 4.1 continued. Since $|f|_{L}^{\frac{2(n-1)}{n-2}}{ }_{\left(\mathbb{R}^{n-1}\right)}=1$ and it is strictly decreasing along the radial direction, we see $0<f(\xi)<\infty$ for $\xi \neq 0$. Note that since $f$ satisfies the Euler-Lagrange equation, it is defined everywhere instead of almost everywhere. It follows from Proposition 4.1 that $f(\xi)=\left(c_{1}|\xi|^{2}+c_{2}\right)^{-\frac{n-2}{2}}$ for some $c_{1}, c_{2}>0$ (note that $f$ can not be a constant function and the scalar multiple of $|\xi|^{2-n}$ is ruled out by the integrability). A simple change of variable shows

$$
1=\int_{\mathbb{R}^{n-1}} f(\xi)^{\frac{2(n-1)}{n-2}} d \xi=\left(c_{1} c_{2}\right)^{-\frac{n-1}{2}} \int_{\mathbb{R}^{n-1}}\left(1+|\xi|^{2}\right)^{-(n-1)} d \xi
$$

Hence $c_{1} c_{2}=c(n)$. It follows that for some $\lambda>0, f(\xi)=c(n)\left(\frac{\lambda}{\lambda^{2}+|\xi|^{2}}\right)^{\frac{n-2}{2}}$. Let $e_{n}=(0, \cdots, 0,1)$. Since $u(x)=\left|x+e_{n}\right|^{2-n}$ is a bounded harmonic function on $\overline{\mathbb{R}_{+}^{n}}$ and $u(\xi, 0)=\left(1+|\xi|^{2}\right)^{-\frac{n-2}{2}}$, we see

$$
P\left(\left(1+|\xi|^{2}\right)^{-\frac{n-2}{2}}\right)(x)=\left|x+e_{n}\right|^{2-n} .
$$

By the dilation invariance

$$
c_{n, \frac{2(n-1)}{n-2}}=\frac{|| x+\left.\left.e_{n}\right|^{2-n}\right|_{L^{\frac{2 n}{n-2}}\left(\mathbb{R}_{+}^{n}\right)}}{\left|\left(1+|\xi|^{2}\right)^{-\frac{n-2}{2}}\right|_{L^{\frac{2(n-1)}{n-2}}\left(\mathbb{R}^{n-1}\right)}}=n^{-\frac{n-2}{2(n-1)}} \omega_{n}^{-\frac{n-2}{2 n(n-1)}} .
$$

For the case when $p=\frac{2(n-1)}{n}$, we know any maximizer after multiplying by a constant will be nonnegative and satisfy

$$
f(\xi)^{\frac{n-2}{n}}=\int_{\mathbb{R}_{+}^{n}} P(x, \xi)(P f)(x) d x=c(n) \int_{\mathbb{R}^{n-1}} \frac{f(\zeta)}{|\xi-\zeta|^{n-2}} d \zeta .
$$

Let $g(\xi)=f(\xi)^{\frac{n-2}{n}}$, then $g \in L^{\frac{2(n-1)}{n-2}}\left(\mathbb{R}^{n-1}\right)$ and

$$
g(\xi)=c(n) \int_{\mathbb{R}^{n-1}} \frac{g(\zeta)^{\frac{(n-1)+1}{(n-1)-1}}}{|\xi-\zeta|^{(n-1)-1}} d \zeta
$$

It follows from [CLO2, theorem 1] or $\left[\mathrm{L}\right.$ that for some $\lambda>0$ and $\xi_{0} \in \mathbb{R}^{n-1}$, we have

Hence

$$
g(\xi)=c(n)\left(\frac{\lambda}{\lambda^{2}+\left|\xi-\xi_{0}\right|^{2}}\right)^{\frac{n-2}{2}} .
$$

$$
f(\xi)=c(n)\left(\frac{\lambda}{\lambda^{2}+\left|\xi-\xi_{0}\right|^{2}}\right)^{\frac{n}{2}}
$$


Since $u(x)=\frac{x_{n}+1}{\left|x+e_{n}\right|^{n}}$ is a bounded harmonic function on $\overline{\mathbb{R}_{+}^{n}}$ and $u(\xi, 0)=\left(1+|\xi|^{2}\right)^{-\frac{n}{2}}$, we see

$$
P\left(\left(1+|\xi|^{2}\right)^{-\frac{n}{2}}\right)(x)=\frac{x_{n}+1}{\left|x+e_{n}\right|^{n}} .
$$

By the dilation invariance

$$
c_{n, \frac{2(n-1)}{n}}=\frac{\left|\frac{x_{n}+1}{\left|x+e_{n}\right|^{n}}\right|_{L^{2}\left(\mathbb{R}_{+}^{n}\right)}}{\left|\left(1+|\xi|^{2}\right)^{-\frac{n}{2}}\right|_{L \frac{2(n-1)}{n}\left(\mathbb{R}^{n-1}\right)}}=\frac{1}{\sqrt{2(n-2)} \sqrt[4]{\pi}}\left(\frac{(n-2) !}{\Gamma\left(\frac{n-1}{2}\right)}\right)^{\frac{1}{2(n-1)}} .
$$

As a final note, we point out the similar statement to Proposition4.1]in dimension one.

Proposition 4.2. Assume $u \in C^{3}(\mathbb{R}), u>0, \alpha \in \mathbb{R}$ such that for any $y \in \mathbb{R}$, $|x|^{\alpha} u\left(\frac{1}{x}+y\right)$ is symmetric with respect to some point, then for some $a \geq 0, b>0$ and $x_{0} \in \mathbb{R}$, we have $u(x)=\left[a\left(x-x_{0}\right)^{2}+b\right]^{\alpha / 2}$.

Proof. Assume $|x|^{\alpha} u\left(\frac{1}{x}+y\right)$ is symmetric with respect to $z=z(y)$, then

$$
|x|^{\alpha} u\left(\frac{1}{x}+y\right)=|2 z-x|^{\alpha} u\left(\frac{1}{2 z-x}+y\right) .
$$

Replace $x$ by $x^{-1}$, we see

$$
u(x+y)=|1-2 z x|^{\alpha} u\left(y-\frac{x}{1-2 z x}\right) .
$$

Calculation shows

$$
\begin{aligned}
& |1-2 z x|^{\alpha} u\left(y-\frac{x}{1-2 z x}\right) \\
& =u(y)-\left(u^{\prime}(y)+2 \alpha z u(y)\right) x+\left(\frac{u^{\prime \prime}(y)}{2}+2(\alpha-1) z u^{\prime}(y)+2 \alpha(\alpha-1) z^{2} u(y)\right) x^{2} \\
& -\left[\frac{u^{\prime \prime \prime}(y)}{6}+(\alpha-2) z u^{\prime \prime}(y)+2(\alpha-1)(\alpha-2) z^{2} u^{\prime}(y)+\frac{4}{3} \alpha(\alpha-1)(\alpha-2) z^{3} u(y)\right] x^{3} \\
& +o\left(x^{3}\right) .
\end{aligned}
$$

Comparing the Taylor expansion coefficients, we see

$$
u^{\prime}(y)=-\alpha z u(y)
$$

and

$\frac{u^{\prime \prime \prime}(y)}{3}+(\alpha-2) z u^{\prime \prime}(y)+2(\alpha-1)(\alpha-2) z^{2} u^{\prime}(y)+\frac{4}{3} \alpha(\alpha-1)(\alpha-2) z^{3} u(y)=0$

If $\alpha=0$, then we see $u^{\prime}=0$ and hence $u$ must be a constant function and we are done. Assume $\alpha \neq 0$, then

$$
z=-\frac{u^{\prime}(y)}{\alpha u(y)}
$$

Plug this in the second equation, we get

$$
u^{2} u^{\prime \prime \prime}+3\left(\frac{2}{\alpha}-1\right) u u^{\prime} u^{\prime \prime}+\left(\frac{2}{\alpha}-1\right)\left(\frac{2}{\alpha}-2\right) u^{\prime 3}=0
$$


Hence

$$
\left(u^{2 / \alpha}\right)^{\prime \prime \prime}=\frac{2}{\alpha} u^{\frac{2}{\alpha}-3}\left[u^{2} u^{\prime \prime \prime}+3\left(\frac{2}{\alpha}-1\right) u u^{\prime} u^{\prime \prime}+\left(\frac{2}{\alpha}-1\right)\left(\frac{2}{\alpha}-2\right) u^{\prime 3}\right]=0 .
$$

The proposition follows.

\section{Regularity of NONNEGATIVE CRITICAL FUnCTIONS}

In this section we will study the regularity issue related to the Euler-Lagrange equation (1.9). Let $f$ be a nonnegative function satisfying (1.9), define $u=P f$, then the single equation becomes an integral system

$$
\begin{aligned}
u(x) & =\int_{\mathbb{R}^{n-1}} P(x, \xi) f(\xi) d \xi, \\
f(\xi)^{p-1} & =\int_{\mathbb{R}_{+}^{n}} P(x, \xi) u(x)^{\frac{n p}{n-1}-1} d x .
\end{aligned}
$$

This system is very similar to the one appeared in the study of the sharp HardyLittlewood-Sobolev inequality ( $\mathrm{Li} 2$, part (ii) of theorem 2.3]). In [ChL, L] the regularity problem for some cases of that system was resolved by a linear approach. In $[\mathrm{Hn}$, a nonlinear approach was introduced to resolve the regularity issue for all the cases. We will apply the nonlinear approach to handle (1.9).

Theorem 5.1. Assume $n \geq 2,1<p<\infty, f \in L_{l o c}^{p}\left(\mathbb{R}^{n-1}\right)$ is nonnegative, not identically zero and it satisfies

$$
f(\xi)^{p-1}=\int_{\mathbb{R}_{+}^{n}} P(x, \xi)(P f)(x)^{\frac{n p}{n-1}-1} d x,
$$

then $f \in C^{\infty}\left(\mathbb{R}^{n-1}\right)$. If we know $f \in L^{p}\left(\mathbb{R}^{n-1}\right)$, then $f(\xi) \rightarrow 0$ as $|\xi| \rightarrow \infty$.

We note that the condition $f \in L_{l o c}^{p}\left(\mathbb{R}^{n-1}\right)$ can not be dropped, since the above equation has $c(n, p)|\xi|^{-\frac{n-1}{p}}$ as a singular solution. To prove this theorem, we first derive some local regularity results for some integral inequalities. According to the range of $p$, we need two local results stated in Proposition 5.1 and Proposition 5.2 below. The two propositions are of the same nature as [Hn, proposition 2.1] and [L, theorem 1.3].

Proposition 5.1. Given $n \geq 2,1<a, b \leq \infty, 1 \leq r<\infty, \frac{n}{n-1}<p<q<\infty$ such that

and

$$
\frac{1}{n}<\frac{r}{q}+\frac{1}{a}<\frac{r}{p}+\frac{1}{a} \leq 1
$$

$$
\frac{n}{r a}+\frac{n-1}{b}=\frac{1}{r}
$$

Denote $B_{R}=B_{R}^{n-1}$ and $B_{R}^{+}=B_{R}^{n} \cap \mathbb{R}_{+}^{n}$. Assume $u, v \in L^{p}\left(B_{R}^{+}\right), U \in L^{a}\left(B_{R}^{+}\right)$, $F \in L^{b}\left(B_{R}\right)$ are all nonnegative functions with $\left.v\right|_{B_{R / 2}^{+}} \in L^{q}\left(B_{R / 2}^{+}\right)$,

$$
|U|_{L^{a}\left(B_{R}^{+}\right)}^{1 / r}|F|_{L^{b}\left(B_{R}\right)} \leq \varepsilon(n, p, q, r, a, b) \text { small }
$$

and

$$
u(x) \leq \int_{B_{R}} P(x, \xi) F(\xi)\left[\int_{B_{R}^{+}} P(y, \xi) U(y) u(y)^{r} d y\right]^{1 / r} d \xi+v(x)
$$


for $x \in B_{R}^{+}$, then we have $\left.u\right|_{B_{R / 4}^{+}} \in L^{q}\left(B_{R / 4}^{+}\right)$and

$$
|u|_{L^{q}\left(B_{R / 4}^{+}\right)} \leq c(n, p, q, r, a, b)\left(R^{\frac{n}{q}-\frac{n}{p}}|u|_{L^{p}\left(B_{R}^{+}\right)}+|v|_{L^{q}\left(B_{R / 2}^{+}\right)}\right) .
$$

Proof. By scaling we may assume $R=1$. First assume we have $u, v \in L^{q}\left(B_{1}^{+}\right)$. Denote

$$
f(\xi)=\int_{B_{1}^{+}} P(x, \xi) U(x) u(x)^{r} d x \text { for } \xi \in B_{1} .
$$

Let $p_{1}$ and $q_{1}$ be the numbers defined by

$$
\frac{n-1}{p_{1}}=\frac{n r}{p}+\frac{n}{a}-1, \quad \frac{n-1}{q_{1}}=\frac{n r}{q}+\frac{n}{a}-1,
$$

then it follows from Proposition 2.2 that

$$
\begin{aligned}
& |f|_{L^{p_{1}\left(B_{1}\right)}} \leq c(n, p, r, a)|U|_{L^{a}\left(B_{1}^{+}\right)}|u|_{L^{p}\left(B_{1}^{+}\right)}^{r}, \\
& |f|_{L^{q_{1}}\left(B_{1}\right)} \leq c(n, q, r, a)|U|_{L^{a}\left(B_{1}^{+}\right)}|u|_{L^{q}\left(B_{1}^{+}\right)}^{r} .
\end{aligned}
$$

Given $0<s<t \leq 1 / 2$. For $x \in B_{s}^{+}$, we have

$$
\begin{aligned}
& u(x) \\
& \leq \int_{B_{\frac{s+t}{2}}} P(x, \xi) F(\xi) f(\xi)^{1 / r} d \xi+\int_{B_{1} \backslash B_{\frac{s+t}{2}}} P(x, \xi) F(\xi) f(\xi)^{1 / r} d \xi+v(x) \\
& \leq \int_{B_{\frac{s+t}{2}}} P(x, \xi) F(\xi) f(\xi)^{1 / r} d \xi+\frac{c(n)}{(t-s)^{n-1}} \int_{B_{1} \backslash B_{\frac{s+t}{2}}} F(\xi) f(\xi)^{1 / r} d \xi+v(x) \\
& \leq \int_{B_{\frac{s+t}{2}}} P(x, \xi) F(\xi) f(\xi)^{1 / r} d \xi+\frac{c(n, p)}{(t-s)^{n-1}}|F|_{L^{b}\left(B_{1}\right)}|f|_{L^{p_{1}\left(B_{1}\right)}}^{1 / r}+v(x) \\
& \leq \int_{B_{\frac{s+t}{2}}} P(x, \xi) F(\xi) f(\xi)^{1 / r} d \xi+\frac{c(n, p, r, a)}{(t-s)^{n-1}|u|_{L^{p}\left(B_{1}^{+}\right)}+v(x) .}
\end{aligned}
$$

Hence

$$
|u|_{L^{q}\left(B_{s}^{+}\right)} \leq c(n, q)|F|_{L^{b}\left(B_{1}\right)}|f|_{L^{q_{1}}\left(B_{\frac{s+t}{2}}\right)}^{1 / r}+\frac{c(n, p, q, r, a)}{(t-s)^{n-1}}|u|_{L^{p}\left(B_{1}^{+}\right)}+|v|_{L^{q}\left(B_{1 / 2}^{+}\right)} .
$$

On the other hand, for $\xi \in B_{\frac{s+t}{2}}$, we have

$$
\begin{aligned}
f(\xi) & =\int_{B_{t}^{+}} P(x, \xi) U(x) u(x)^{r} d x+\int_{B_{1}^{+} \backslash B_{t}^{+}} P(x, \xi) U(x) u(x)^{r} d x \\
& \leq \int_{B_{t}^{+}} P(x, \xi) U(x) u(x)^{r} d x+\frac{c(n)}{(t-s)^{n-1}} \int_{B_{1}^{+} \backslash B_{t}^{+}} U(x) u(x)^{r} d x \\
& \leq \int_{B_{t}^{+}} P(x, \xi) U(x) u(x)^{r} d x+\frac{c(n, p, r, a)}{(t-s)^{n-1}}|U|_{L^{a}\left(B_{1}^{+}\right)}|u|_{L^{p}\left(B_{1}^{+}\right)}^{r} .
\end{aligned}
$$

It follows from Proposition 2.2 that

$$
|f|_{L^{q_{1}}\left(B_{\frac{s+t}{2}}\right)} \leq c(n, q, r, a)|U|_{L^{a}\left(B_{1}^{+}\right)}|u|_{L^{q}\left(B_{t}^{+}\right)}^{r}+\frac{c(n, p, q, r, a)}{(t-s)^{n-1}}|U|_{L^{a}\left(B_{1}^{+}\right)}|u|_{L^{p}\left(B_{1}^{+}\right)}^{r} .
$$


Combining the two inequalities together, we see

$$
|u|_{L^{q}\left(B_{s}^{+}\right)} \leq \frac{1}{2}|u|_{L^{q}\left(B_{t}^{+}\right)}+\frac{c(n, p, q, r, a)}{(t-s)^{n-1}}|u|_{L^{p}\left(B_{1}^{+}\right)}+|v|_{L^{q}\left(B_{1 / 2}^{+}\right)}
$$

if $\varepsilon$ is small enough. It follows from the usual iteration procedure ( $\mathrm{HL}$, lemma 4.3 on p.75]) that

$$
|u|_{L^{q}\left(B_{1 / 4}^{+}\right)} \leq c(n, p, q, r, a)\left(|u|_{L^{p}\left(B_{1}^{+}\right)}+|v|_{L^{q}\left(B_{1 / 2}^{+}\right)}\right) .
$$

To prove the full proposition, we note that there exists a function $0 \leq \eta(x) \leq 1$ such that

$$
u(x)=\eta(x) \int_{B_{1}} P(x, \xi) F(\xi)\left[\int_{B_{1}^{+}} P(y, \xi) U(y) u(y)^{r} d y\right]^{1 / r} d \xi+\eta(x) v(x) .
$$

We may define a map $T$ by

$$
T(\varphi)(x)=\eta(x) \int_{B_{1}} P(x, \xi) F(\xi)\left[\int_{B_{1}^{+}} P(y, \xi) U(y)|\varphi(y)|^{r} d y\right]^{1 / r} d \xi .
$$

Note that we have

$$
\begin{aligned}
|T(\varphi)|_{L^{p}\left(B_{1}^{+}\right)} & \leq c(n, p, r, a, b)|U|_{L^{a}\left(B_{1}^{+}\right)}^{1 / r}|F|_{L^{b}\left(B_{1}\right)}|\varphi|_{L^{p}\left(B_{1}\right)} \leq \frac{1}{2}|\varphi|_{L^{p}\left(B_{1}\right)}, \\
|T(\varphi)|_{L^{q}\left(B_{1}^{+}\right)} & \leq c(n, q, r, a, b)|U|_{L^{a}\left(B_{1}^{+}\right)}^{1 / r}|F|_{L^{b}\left(B_{1}\right)}|\varphi|_{L^{q}\left(B_{1}\right)} \leq \frac{1}{2}|\varphi|_{L^{q}\left(B_{1}\right)}
\end{aligned}
$$

if $\varepsilon$ is small enough. Moreover, for $\varphi, \psi \in L^{p}\left(B_{1}^{+}\right)$, it follows from Minkowski inequality that

$$
|T(\varphi)(x)-T(\psi)(x)| \leq T(|\varphi-\psi|)(x) \text { for } x \in B_{1}^{+},
$$

hence

$$
|T(\varphi)-T(\psi)|_{L^{p}\left(B_{1}^{+}\right)} \leq|T(|\varphi-\psi|)|_{L^{p}\left(B_{1}^{+}\right)} \leq \frac{1}{2}|\varphi-\psi|_{L^{p}\left(B_{1}^{+}\right)} .
$$

Similarly we have for any $\varphi, \psi \in L^{q}\left(B_{1}^{+}\right)$,

$$
|T(\varphi)-T(\psi)|_{L^{q}\left(B_{1}^{+}\right)} \leq \frac{1}{2}|\varphi-\psi|_{L^{q}\left(B_{1}^{+}\right)} .
$$

For $k \in \mathbb{N}$, let $v_{k}(x)=\min \{v(x), k\}$, then it follows from contraction mapping theorem that we may find a unique $u_{k} \in L^{q}\left(B_{1}^{+}\right)$such that

$$
\begin{aligned}
& u_{k}(x) \\
& =T\left(u_{k}\right)(x)+\eta(x) v_{k}(x) \\
& =\eta(x) \int_{B_{1}} P(x, \xi) F(\xi)\left[\int_{B_{1}^{+}} P(y, \xi) U(y)\left|u_{k}(y)\right|^{r} d y\right]^{1 / r} d \xi+\eta(x) v_{k}(x) .
\end{aligned}
$$

Applying the apriori estimate to $u_{k}$, we see

$$
\left|u_{k}\right|_{L^{q}\left(B_{1 / 4}^{+}\right)} \leq c(n, p, q, r, a)\left(\left|u_{k}\right|_{L^{p}\left(B_{1}^{+}\right)}+|v|_{L^{q}\left(B_{1 / 2}^{+}\right)}\right) .
$$

Observe that

$$
u(x)=T(u)(x)+\eta(x) v(x)
$$


we see

$$
\begin{aligned}
\left|u_{k}-u\right|_{L^{p}\left(B_{1}^{+}\right)} & \leq\left|T\left(u_{k}\right)-T(u)\right|_{L^{p}\left(B_{1}^{+}\right)}+\left|v_{k}-v\right|_{L^{p}\left(B_{1}^{+}\right)} \\
& \leq \frac{1}{2}\left|u_{k}-u\right|_{L^{p}\left(B_{1}^{+}\right)}+\left|v_{k}-v\right|_{L^{p}\left(B_{1}^{+}\right)} .
\end{aligned}
$$

Hence $\left|u_{k}-u\right|_{L^{p}\left(B_{1}^{+}\right)} \leq 2\left|v_{k}-v\right|_{L^{p}\left(B_{1}^{+}\right)} \rightarrow 0$ as $k \rightarrow \infty$. Take a limit process in the apriori estimate, we get the proposition.

The other local regularity result is

Proposition 5.2. Given $n \geq 2,1<a, b \leq \infty, 1 \leq r<\infty, 1<p<q<\infty$ such that

and

$$
0<\frac{r}{p}+\frac{1}{a}<1
$$

$$
\frac{n-1}{r a}+\frac{n}{b}=1 \text {. }
$$

Denote $B_{R}=B_{R}^{n-1}$ and $B_{R}^{+}=B_{R}^{n} \cap \mathbb{R}_{+}^{n}$. Assume $f, g \in L^{p}\left(B_{R}\right), F \in L^{a}\left(B_{R}\right)$, $U \in L^{b}\left(B_{R}^{+}\right)$are all nonnegative functions with $\left.g\right|_{B_{R / 2}} \in L^{q}\left(B_{R / 2}\right)$,

$$
|F|_{L^{a}\left(B_{R}\right)}^{1 / r}|U|_{L^{b}\left(B_{R}^{+}\right)} \leq \varepsilon(n, p, q, r, a, b) \text { small }
$$

and

$$
f(\xi) \leq \int_{B_{R}^{+}} P(x, \xi) U(x)\left[\int_{B_{R}} P(x, \zeta) F(\zeta) f(\zeta)^{r} d \zeta\right]^{1 / r} d x+g(\xi)
$$

for $\xi \in B_{R}$, then we have $\left.f\right|_{B_{R / 4}} \in L^{q}\left(B_{R / 4}\right)$ and

$$
|f|_{L^{q}\left(B_{R / 4}\right)} \leq c(n, p, q, r, a, b)\left(R^{\frac{n-1}{q}-\frac{n-1}{p}}|f|_{L^{p}\left(B_{R}\right)}+|g|_{L^{q}\left(B_{R / 2}\right)}\right) .
$$

Proof. By scaling, we may assume $R=1$. First assume we have $f, g \in L^{q}\left(B_{1}\right)$. Define

$$
u(x)=\int_{B_{1}} P(x, \xi) F(\xi) f(\xi)^{r} d \xi
$$

for $x \in B_{1}^{+}$. Let $p_{1}$ and $q_{1}$ be the numbers given by

$$
\frac{n}{p_{1}}=\frac{n-1}{a}+\frac{(n-1) r}{p}, \quad \frac{n}{q_{1}}=\frac{n-1}{a}+\frac{(n-1) r}{q} .
$$

It follows from Proposition 2.1 that

$$
\begin{aligned}
|u|_{L^{p_{1}}\left(B_{1}^{+}\right)} & \leq c(n, p, r, a)|F|_{L^{a}\left(B_{1}\right)}|f|_{L^{p}\left(B_{1}\right)}^{r}, \\
|u|_{L^{q_{1}}\left(B_{1}^{+}\right)} & \leq c(n, q, r, a)|F|_{L^{a}\left(B_{1}\right)}|f|_{L^{q}\left(B_{1}\right)}^{r} .
\end{aligned}
$$

Given $0<s<t \leq 1 / 2$. For $\xi \in B_{s}$, a similar calculation as in the proof of Proposition 5.1 shows

$$
f(\xi) \leq \int_{B_{\frac{s+t}{2}}^{+}} P(x, \xi) U(x) u(x)^{1 / r} d x+\frac{c(n, p, r, a)}{(t-s)^{n-1}}|f|_{L^{p}\left(B_{1}\right)}+g(\xi) .
$$

Hence

$$
|f|_{L^{q}\left(B_{s}\right)} \leq c(n, q, r, b)|U|_{L^{b}\left(B_{1}^{+}\right)}|u|_{L^{q_{1}}\left(B_{\frac{s+t}{2}}^{+/ r}\right)}^{1 /(t-s)^{n-1}}|f|_{L^{p}\left(B_{1}\right)}+|g|_{L^{q}\left(B_{1 / 2}\right)} .
$$


On the other hand, for $x \in B_{\frac{s+t}{2}}^{+}$, we have

$$
u(x) \leq \int_{B_{t}} P(x, \xi) F(\xi) f(\xi)^{r} d \xi+\frac{c(n, p, r, a)}{(t-s)^{n-1}}|F|_{L^{a}\left(B_{1}\right)}|f|_{L^{p}\left(B_{1}\right)}^{r} .
$$

Hence

$$
|u|_{L^{q_{1}}\left(B_{\frac{s+t}{2}}^{+}\right)} \leq c(n, q, r, a)|F|_{L^{a}\left(B_{1}\right)}|f|_{L^{q}\left(B_{t}\right)}^{r}+\frac{c(n, p, q, r, a)}{(t-s)^{n-1}}|F|_{L^{a}\left(B_{1}\right)}|f|_{L^{p}\left(B_{1}\right)}^{r} .
$$

Combining the two inequalities together, we see

$$
|f|_{L^{q}\left(B_{s}\right)} \leq \frac{1}{2}|f|_{L^{q}\left(B_{t}\right)}+\frac{c(n, p, q, r, a, b)}{(t-s)^{n-1}}|f|_{L^{p}\left(B_{1}\right)}+|g|_{L^{q}\left(B_{1 / 2}\right)}
$$

when $\varepsilon$ is small enough. This implies

$$
|f|_{L^{q}\left(B_{1 / 4}\right)} \leq c(n, p, q, r, a, b)\left(|f|_{L^{p}\left(B_{1}\right)}+|g|_{L^{q}\left(B_{1 / 2}\right)}\right) \text {. }
$$

With this apriori estimate at hands, we may proceed in the same way as the proof of Proposition 5.1 to get the full conclusion.

Now we are ready to derive the main results of this section.

Proof of Theorem 5.1. Let $p_{0}=\frac{1}{p-1}, f_{0}(\xi)=f(\xi)^{p-1}, u_{0}(x)=(P f)(x)$, then $0<p_{0}<\infty, f_{0} \in L_{l o c}^{p_{0}+1}\left(\mathbb{R}^{n-1}\right)$ and

$$
u_{0}(x)=\int_{\mathbb{R}^{n-1}} P(x, \xi) f_{0}(\xi)^{p_{0}} d \xi, \quad f_{0}(\xi)=\int_{\mathbb{R}_{+}^{n}} P(x, \xi) u_{0}(x)^{\frac{p_{0}+n}{(n-1) p_{0}}} d x .
$$

For $R>0$, we write $B_{R}=B_{R}^{n-1}, B_{R}^{+}=B_{R}^{n} \cap \mathbb{R}_{+}^{n}$ and

$$
\begin{aligned}
& u_{R}(x)=\int_{\mathbb{R}^{n-1} \backslash B_{R}} P(x, \xi) f_{0}(\xi)^{p_{0}} d \xi \\
& f_{R}(\xi)=\int_{\mathbb{R}_{+}^{n} \backslash B_{R}^{+}} P(x, \xi) u_{0}(x)^{\frac{p_{0}+n}{(n-1) p_{0}}} d x .
\end{aligned}
$$

First we want to show $u_{0} \in L_{l o c}^{\frac{n\left(p_{0}+1\right)}{(n-1) p_{0}}}\left(\overline{\mathbb{R}}_{+}^{n}\right)$ and $u_{R} \in L^{\frac{n\left(p_{0}+1\right)}{(n-1) p_{0}}}\left(B_{R}^{+}\right) \cap L_{l o c}^{\infty}\left(B_{R}^{+} \cup B_{R}^{n-1}\right)$. Indeed, since $f_{0} \in L_{\text {loc }}^{p_{0}+1}\left(\mathbb{R}^{n-1}\right)$, we see $f_{0}<\infty$ a.e. on $\mathbb{R}^{n-1}$. This implies $u_{0}<\infty$ a.e. on $\mathbb{R}_{+}^{n}$. Hence there exists an $x_{0} \in B_{R}^{+}$such that $u_{0}\left(x_{0}\right)<\infty$. It follows that $\int_{\mathbb{R}^{n-1} \backslash B_{R}} \frac{f_{0}(\xi)^{p_{0}}}{\left(\left|x_{0}^{\prime}-\xi\right|^{2}+x_{0, n}^{2}\right)^{n / 2}} d \xi<\infty$ and $\int_{\mathbb{R}^{n-1} \backslash B_{R}} \frac{f_{0}(\xi)^{p_{0}}}{|\xi|^{n}} d \xi<\infty$. For $0<\theta<1$, $x \in B_{\theta R}^{+}$, we have

$$
u_{R}(x)=\int_{\mathbb{R}^{n-1} \backslash B_{R}} P(x, \xi) f_{0}(\xi)^{p_{0}} d \xi \leq \frac{c(n) R}{(1-\theta)^{n}} \int_{\mathbb{R}^{n-1} \backslash B_{R}} \frac{f_{0}(\xi)^{p_{0}}}{|\xi|^{n}} d \xi .
$$

It follows that $u_{R} \in L_{l o c}^{\infty}\left(B_{R}^{+} \cup B_{R}^{n-1}\right)$. Since $\int_{B_{R}} P(\cdot, \xi) f_{0}(\xi)^{p_{0}} d \xi \in L^{\frac{n\left(p_{0}+1\right)}{(n-1) p_{0}}}\left(\mathbb{R}_{+}^{n}\right)$, we know $u_{0} \in L_{l o c}^{\frac{n\left(p_{0}+1\right)}{(n-1) p_{0}}}\left(B_{R}^{+} \cup B_{R}^{n-1}\right)$. By choosing $R$ arbitrarily large, we deduce that $u_{0} \in L_{l o c}^{\frac{n\left(p_{0}+1\right)}{(n-1) p_{0}}}\left(\overline{\mathbb{R}}_{+}^{n}\right)$ and hence $u_{R} \in L^{\frac{n\left(p_{0}+1\right)}{(n-1) p_{0}}}\left(B_{R}^{+}\right)$. 
Next we want to show $f_{R} \in L^{p_{0}+1}\left(B_{R}\right) \cap L_{\text {loc }}^{\infty}\left(B_{R}\right)$. Indeed we may find $\xi_{0} \in B_{R}$ such that $\int_{\mathbb{R}_{+}^{n}} P\left(x, \xi_{0}\right) u_{0}(x)^{\frac{p_{0}+n}{(n-1) p_{0}}} d x<\infty$. This implies

$$
\int_{\mathbb{R}_{+}^{n} \backslash B_{R}^{+}} \frac{x_{n}}{\left(\left|x^{\prime}-\xi_{0}\right|^{2}+x_{n}^{2}\right)^{n / 2}} u_{0}(x)^{\frac{p_{0}+n}{(n-1) p_{0}}} d x<\infty
$$

and hence $\int_{\mathbb{R}_{+}^{n} \backslash B_{R}^{+}} \frac{x_{n}}{|x|^{n}} u_{0}(x)^{\frac{p_{0}+n}{(n-1) p_{0}}} d x<\infty$. For $0<\theta<1, \xi \in B_{\theta R}$, we have

$$
f_{R}(\xi)=\int_{\mathbb{R}_{+}^{n} \backslash B_{R}^{+}} P(x, \xi) u_{0}(x)^{\frac{p_{0}+n}{(n-1) p_{0}}} d x \leq \frac{c(n)}{(1-\theta)^{n}} \int_{\mathbb{R}_{+}^{n} \backslash B_{R}^{+}} \frac{x_{n}}{|x|^{n}} u_{0}(x)^{\frac{p_{0}+n}{(n-1) p_{0}}} d x
$$

and hence $f_{R} \in L_{l o c}^{\infty}\left(B_{R}\right)$. To prove the regularity of $f$, we discuss two cases.

Case 5.1. $0<p_{0} \leq \frac{n}{n-1}$.

In this case, we have $\frac{p_{0}+n}{(n-1) p_{0}}>1$. Fix a number $r$ such that

$$
1 \leq r<\frac{p_{0}+n}{(n-1) p_{0}} \text { and } r>\frac{1}{p_{0}}
$$

then

$$
f_{0}(\xi)^{1 / r} \leq\left(\int_{B_{R}^{+}} P(x, \xi) u_{0}(x)^{\frac{p_{0}+n}{(n-1) p_{0}}} d x\right)^{1 / r}+f_{R}(\xi)^{1 / r}
$$

Hence

$$
\begin{aligned}
& u_{0}(x) \\
& =\int_{B_{R}} P(x, \xi) f_{0}(\xi)^{p_{0}-r^{-1}} f_{0}(\xi)^{1 / r} d \xi+u_{R}(x) \\
& \leq \int_{B_{R}} P(x, \xi) f_{0}(\xi)^{p_{0}-r^{-1}}\left(\int_{B_{R}^{+}} P(y, \xi) u_{0}(y)^{\frac{p_{0}+n}{(n-1) p_{0}}-r} u_{0}(y)^{r} d y\right)^{1 / r} d \xi+v_{R}(x),
\end{aligned}
$$

here

$$
v_{R}(x)=\int_{B_{R}} P(x, \xi) f_{0}(\xi)^{p_{0}-r^{-1}} f_{R}(\xi)^{1 / r} d \xi+u_{R}(x) .
$$

Since $f_{R} \in L^{p_{0}+1}\left(B_{R}\right)$, we see $v_{R} \in L^{\frac{n\left(p_{0}+1\right)}{(n-1) p_{0}}}\left(B_{R}^{+}\right)$. On the other hand, for $0<$ $\theta<1, x \in B_{\theta R}^{+}$, we have

$$
\begin{aligned}
& \int_{B_{R}} P(x, \xi) f_{0}(\xi)^{p_{0}-r^{-1}} f_{R}(\xi)^{1 / r} d \xi \\
& \leq\left|f_{R}\right|_{L^{\infty}\left(B_{\frac{1+\theta}{2} R}\right)} \int_{B_{\frac{1+\theta}{2} R}} P(x, \xi) f_{0}(\xi)^{p_{0}-r^{-1}} d \xi \\
& +\frac{c(n)}{(1-\theta)^{n} R^{n-1}} \int_{B_{R} \backslash B_{\frac{1+\theta}{2} R}} f_{0}(\xi)^{p_{0}-r^{-1}} f_{R}(\xi)^{1 / r} d \xi \\
& \leq\left|f_{R}\right|_{L^{\infty}\left(B_{\frac{1+\theta}{2} R}^{1 / r}\right)} \int_{B_{\frac{1+\theta}{2} R}} P(x, \xi) f_{0}(\xi)^{p_{0}-r^{-1}} d \xi+\frac{c\left(n, p_{0}\right)}{(1-\theta)^{n} R^{\frac{(n-1) p_{0}}{p_{0}+1}}}\left|f_{0}\right|_{L^{p_{0}+1}\left(B_{R}\right)}^{p_{0}},
\end{aligned}
$$


hence $v_{R} \in L_{l o c}^{\frac{n\left(p_{0}+1\right)}{(n-1)\left(p_{0}-r^{-1}\right)}}\left(B_{R}^{+} \cup B_{R}^{n-1}\right)$. Let

$$
a=\frac{n\left(p_{0}+1\right)}{p_{0}+n-(n-1) p_{0} r}, \quad b=\frac{\left(p_{0}+1\right) r}{p_{0} r-1} .
$$

Then $\frac{n}{r a}+\frac{n-1}{b}=\frac{1}{r}$ and

$$
\frac{r}{\frac{n\left(p_{0}+1\right)}{(n-1) p_{0}}}+\frac{1}{a}=\frac{p_{0}+n}{n\left(p_{0}+1\right)}<1
$$

For $\frac{n\left(p_{0}+1\right)}{(n-1) p_{0}}<q<\frac{n\left(p_{0}+1\right)}{(n-1)\left(p_{0}-r^{-1}\right)}$, we have $\frac{r}{q}+\frac{1}{a}>\frac{1}{n}$. It follows from Proposition 5.1 that $\left.u_{0}\right|_{B_{R / 4}^{+}} \in L^{q}\left(B_{R / 4}^{+}\right)$. This implies

$$
f_{0}(\xi)=\int_{B_{R / 4}^{+}} P(x, \xi) u_{0}(x)^{\frac{p_{0}+n}{(n-1) p_{0}}} d x+f_{R / 4}(\xi) \leq c(n, q)\left|u_{0}\right|_{L^{q}\left(B_{R / 4}^{+}\right)}^{\frac{p_{0}+n}{(n-1) p_{0}}}+f_{R / 4}(\xi)
$$

when $q>\frac{n\left(p_{0}+n\right)}{(n-1) p_{0}}$. Such a choice of $q$ is possible since $\frac{n\left(p_{0}+1\right)}{(n-1)\left(p_{0}-r^{-1}\right)}>\frac{n\left(p_{0}+n\right)}{(n-1) p_{0}}$. In particular, we see $\left.f_{0}\right|_{B_{R / 8}} \in L^{\infty}\left(B_{R / 8}\right)$. Since every point may be viewed as a center, we see $f_{0} \in L_{l o c}^{\infty}\left(\mathbb{R}^{n-1}\right)$ and hence $u_{0} \in L_{l o c}^{\infty}\left(\overline{\mathbb{R}}_{+}^{n}\right)$. For any $R>0$, since

$$
\int_{\mathbb{R}^{n-1} \backslash B_{R}} \frac{f_{0}(\xi)^{p_{0}}}{|\xi|^{n}} d \xi<\infty \text { and } \int_{\mathbb{R}_{+}^{n} \backslash B_{R}^{+}} \frac{x_{n}}{|x|^{n}} u_{0}(x)^{\frac{p_{0}+n}{(n-1) p_{0}}} d x<\infty
$$

we see $u_{R} \in C^{\infty}\left(B_{R}^{+} \cup B_{R}^{n-1}\right)$ and $f_{R} \in C^{\infty}\left(B_{R}\right)$. It follows that $f_{0} \in C_{\text {loc }}^{\alpha}\left(\mathbb{R}^{n-1}\right)$ for $0<\alpha<1$. In particular, $f_{0}(\xi)>0$ for any $\xi \in \mathbb{R}^{n-1}$. This implies $u_{0} \in$ $C_{\text {loc }}^{\alpha}\left(\overline{\mathbb{R}}_{+}^{n}\right)$ for any $0<\alpha<1$. Using the fact $\partial_{2} \log |x|=x_{2}|x|^{-2}$ when $n=2$, $\partial_{n}|x|^{2-n}=(2-n) x_{n}|x|^{-n}$ when $n \geq 3$ and the standard potential theory in GT, chapter 4], it follows from bootstrap method that both $u_{0}$ and $f_{0}$ are smooth. If $f \in L^{p}\left(\mathbb{R}^{n-1}\right)$, then $f_{0} \in L^{p_{0}+1}\left(\mathbb{R}^{n-1}\right)$ and $u_{0} \in L^{\frac{n\left(p_{0}+1\right)}{(n-1) p_{0}}}\left(\mathbb{R}_{+}^{n}\right)$. If we go back to the proof with this fact and apply Holder inequality when necessary, we will get $f_{0} \in L^{\infty}\left(\mathbb{R}^{n-1}\right)$ and $u_{0} \in L^{\infty}\left(\mathbb{R}_{+}^{n}\right)$. This implies $u_{0}^{\frac{p_{0}+n}{(n-1) p_{0}}} \in L^{s}\left(\mathbb{R}_{+}^{n}\right)$ for $\frac{n\left(p_{0}+1\right)}{p_{0}+n} \leq s \leq \infty$. Denote

$$
\begin{aligned}
& U=\frac{x_{n}}{|x|^{n}} *\left(u_{0}^{\frac{p_{0}+n}{(n-1) p_{0}}} \chi_{\mathbb{R}_{+}^{n}}\right) \\
& =\left(\frac{x_{n}}{|x|^{n}} \chi_{B_{1}^{n}}\right) *\left(u_{0}^{\frac{p_{0}+n}{(n-1) p_{0}}} \chi_{\mathbb{R}_{+}^{n}}\right)+\left(\frac{x_{n}}{|x|^{n}} \chi_{\mathbb{R}^{n} \backslash B_{1}^{n}}\right) *\left(u_{0}^{\frac{p_{0}+n}{(n-1) p_{0}}} \chi_{\mathbb{R}_{+}^{n}}\right) \text {. }
\end{aligned}
$$

Since $\frac{x_{n}}{|x|^{n}} \chi_{B_{1}^{n}} \in L^{\frac{n}{n-1}-\varepsilon}\left(\mathbb{R}^{n}\right), \frac{x_{n}}{|x|^{n}} \chi_{\mathbb{R}^{n} \backslash B_{1}^{n}} \in L^{\frac{n}{n-1}+\varepsilon}\left(\mathbb{R}^{n}\right)$ and $\frac{n\left(p_{0}+1\right)}{p_{0}+n}<n$, we see $U$ is continuous and $U(x) \rightarrow 0$ as $|x| \rightarrow \infty$. It follows from $f_{0}=\left.c(n) U\right|_{\mathbb{R}^{n-1}}$ that $f_{0}(\xi) \rightarrow 0$ as $|\xi| \rightarrow \infty$.

Case 5.2. $\frac{n}{n-1} \leq p_{0}<\infty$.

In this case, we fix a number $r$ such that

$$
1 \leq r \leq p_{0} \text { and } r \geq \frac{(n-1) p_{0}}{p_{0}+n}
$$


then

$$
u_{0}(x)^{1 / r} \leq\left(\int_{B_{R}} P(x, \xi) f_{0}(\xi)^{p_{0}} d \xi\right)^{1 / r}+u_{R}(x)^{1 / r}
$$

Hence

$$
f_{0}(\xi) \leq \int_{B_{R}^{+}} P(x, \xi) u_{0}(x)^{\frac{p_{0}+n}{(n-1) p_{0}}-r^{-1}}\left(\int_{B_{R}} P(x, \zeta) f_{0}(\zeta)^{p_{0}-r} f_{0}(\zeta)^{r} d \zeta\right)^{1 / r} d x+g_{R}(\xi),
$$

here

$$
g_{R}(\xi)=\int_{B_{R}^{+}} P(x, \xi) u_{0}(x)^{\frac{p_{0}+n}{(n-1) p_{0}}-r^{-1}} u_{R}(x)^{1 / r} d x+f_{R}(\xi) .
$$

Since $u_{R} \in L^{\frac{n\left(p_{0}+1\right)}{(n-1) p_{0}}}\left(B_{R}^{+}\right)$, we see $g_{R} \in L^{p_{0}+1}\left(B_{R}\right)$. On the other hand, for $0<$ $\theta<1, \xi \in B_{\theta R}$, we have

$$
\begin{aligned}
& \int_{B_{R}^{+}} P(x, \xi) u_{0}(x)^{\frac{p_{0}+n}{(n-1) p_{0}}-r^{-1}} u_{R}(x)^{1 / r} d x \\
& \leq\left|u_{R}\right|_{L^{\infty}\left(B_{\frac{1+\theta}{2} R}^{+}\right)}^{1 / r} \int_{B_{\frac{1+\theta}{2} R}^{+}} P(x, \xi) u_{0}(x)^{\frac{p_{0}+n}{(n-1) p_{0}}-r^{-1}} d x \\
& +\frac{c(n)}{(1-\theta)^{n} R^{n-1}} \int_{B_{R}^{+} \backslash B_{\frac{1+\theta}{2} R}^{+}} u_{0}(x)^{\frac{p_{0}+n}{(n-1) p_{0}}-r^{-1}} u_{R}(x)^{1 / r} d x \\
& \leq\left|u_{R}\right|_{L^{\infty}\left(B_{\frac{1+\theta}{2} R}^{+/ r}\right)}^{1 /{ }^{\infty}} \int_{B_{\frac{1+\theta}{2} R}^{+}} P(x, \xi) u_{0}(x)^{\frac{p_{0}+n}{(n-1) p_{0}}-r^{-1}} d x \\
& +\frac{c\left(n, p_{0}\right)}{(1-\theta)^{n} R^{\frac{n-1}{p_{0}+1}}}\left|u_{0}\right|_{L^{\frac{n\left(p_{0}+1\right)}{(n-1) p_{0}}}}^{\frac{p_{0}+n}{(n-1) p_{0}}}\left(B_{R}^{+}\right),
\end{aligned}
$$

hence $g_{R} \in L_{\text {loc }}^{q}\left(B_{R}\right)$ for any $q<\infty$. Let

$$
a=\frac{p_{0}+1}{p_{0}-r}, \quad b=\frac{n\left(p_{0}+1\right) r}{\left(p_{0}+n\right) r-(n-1) p_{0}},
$$

then $\frac{n-1}{r a}+\frac{n}{b}=1, \frac{r}{p_{0}+1}+\frac{1}{a}=\frac{p_{0}}{p_{0}+1} \in(0,1)$. For any $p_{0}+1<q<\infty$, it follows from Proposition 5.2 that when $R$ is small enough, we have $f_{0} \in L^{q}\left(B_{R / 4}\right)$. Since every point can be viewed as a center, we see $f_{0} \in L_{l o c}^{q}\left(\mathbb{R}^{n-1}\right)$ and hence $u_{0} \in L_{l o c}^{\frac{n q}{n-1}}\left(\overline{\mathbb{R}}_{+}^{n}\right)$. Using the equations of $f_{0}$ and $u_{0}$, we see $f_{0} \in L_{l o c}^{\infty}\left(\mathbb{R}^{n-1}\right)$ and $u_{0} \in L_{l o c}^{\infty}\left(\overline{\mathbb{R}}_{+}^{n}\right)$. Now the arguments in Case 5.1 tell us $f_{0} \in C^{\infty}\left(\mathbb{R}^{n-1}\right)$ and $u_{0} \in C^{\infty}\left(\overline{\mathbb{R}}_{+}^{n}\right)$, moreover, $f_{0}(\xi) \rightarrow 0$ as $|\xi| \rightarrow \infty$ under the assumption $f \in L^{p}\left(\mathbb{R}^{n-1}\right)$.

\section{RADIAL SYMMETRY OF NONNEGATIVE CRITICAL FUNCTIONS}

In this section we will study the symmetry property of the nonnegative critical functions of the variational problem (1.8). We will show any nonnegative critical functions are radial symmetric with respect to some point. As explained at the beginning of Section [5, (1.9) may be viewed as an integral system which is very similar to the integral systems related to the Hardy-Littlewood-Sobolev inequalities. For the latter one, the radial symmetry of nonnegative solution for some special cases were solved in [CLO1, CLO2, L]. In particular, in CLO2 an integral version 
of the method of moving planes (GNN]) was introduced and later applied in [CLO1] to resolve the symmetry problems for some cases of the integral systems related to Hardy-Littlewood-Sobolev inequalities. In [Hn], some new observations were added and all the cases for the symmetry of the solutions to the systems were resolved. We will apply these new observations to (1.9).

Theorem 6.1. Assume $1<p<\infty, n \geq 2, f \in L^{p}\left(\mathbb{R}^{n-1}\right)$ is nonnegative, not identically zero and it satisfies

$$
f(\xi)^{p-1}=\int_{\mathbb{R}_{+}^{n}} P(x, \xi)(P f)(x)^{\frac{n p}{n-1}-1} d x,
$$

then $f \in C^{\infty}\left(\mathbb{R}^{n-1}\right)$, moreover $f$ is radial symmetric with respect to some point and strictly decreasing along the radial direction.

For the case $n \geq 3, p=\frac{2(n-1)}{n-2}$, the Euler-Lagrange equation has conformal invariance property and we may weaken the assumption a little bit.

Proposition 6.1. Assume $n \geq 3, f \in L_{l o c}^{\frac{2(n-1)}{n-2}}\left(\mathbb{R}^{n-1}\right)$ is nonnegative, not identically zero and it satisfies

$$
f(\xi)^{\frac{n}{n-2}}=\int_{\mathbb{R}_{+}^{n}} P(x, \xi)(P f)(x)^{\frac{n+2}{n-2}} d x
$$

then for some $\lambda>0$ and $\xi_{0} \in \mathbb{R}^{n-1}$, we have

$$
f(\xi)=c(n)\left(\frac{\lambda}{\lambda^{2}+\left|\xi-\xi_{0}\right|^{2}}\right)^{\frac{n-2}{2}} .
$$

In the proof of these symmetry results, we will need the following basic inequality: assume $0<\theta \leq 1, a \geq b \geq 0, c \geq 0$, then

$$
(a+c)^{\theta}-(b+c)^{\theta} \leq a^{\theta}-b^{\theta} .
$$

For $\sigma \in \mathbb{R}^{m}$ and $s>0$, we denote

$$
|\sigma|_{l^{s}}=\left(\sum_{i=1}^{m}\left|\sigma_{i}\right|^{s}\right)^{1 / s}
$$

Proof of Theorem 6.1. By Theorem 5.1 we know $f \in C^{\infty}\left(\mathbb{R}^{n-1}\right)$ and $f(\xi) \rightarrow 0$ as $|\xi| \rightarrow \infty$. Let $q=\frac{1}{p-1}, g(\xi)=f(\xi)^{p-1}, v(x)=(P f)(x)$, then $0<q<\infty$, $g \in L^{q+1}\left(\mathbb{R}^{n-1}\right), v \in L^{\frac{n(q+1)}{(n-1) q}}\left(\mathbb{R}_{+}^{n}\right)$ and

$$
v(x)=\int_{\mathbb{R}^{n-1}} P(x, \xi) g(\xi)^{q} d \xi, \quad g(\xi)=\int_{\mathbb{R}_{+}^{n}} P(x, \xi) v(x)^{\frac{q+n}{(n-1) q}} d x .
$$

For $\lambda \in \mathbb{R}$, denote

$$
H_{\lambda}=\left\{\xi \in \mathbb{R}^{n-1}: \xi_{1}<\lambda\right\}, \quad Q_{\lambda}=\left\{x \in \mathbb{R}_{+}^{n}: x_{1}<\lambda\right\} .
$$

For $\xi \in \mathbb{R}^{n-1}, \xi=\left(\xi_{1}, \xi^{\prime \prime}\right)$, denote $\xi_{\lambda}=\left(2 \lambda-\xi_{1}, \xi^{\prime \prime}\right)$. For $x \in \mathbb{R}^{n}, x=\left(x_{1}, x^{\prime \prime}\right)$, denote $x_{\lambda}=\left(2 \lambda-x_{1}, x^{\prime \prime}\right)$. Define $g_{\lambda}(\xi)=g\left(\xi_{\lambda}\right), v_{\lambda}(x)=v\left(x_{\lambda}\right)$ and

$$
\mathcal{B}_{\lambda}^{g}=\left\{\xi \in H_{\lambda}: g_{\lambda}(\xi)>g(\xi)\right\}, \quad \mathcal{B}_{\lambda}^{v}=\left\{x \in Q_{\lambda}: v_{\lambda}(x)>v(x)\right\} .
$$


By a simple change of variable, we see

$$
\begin{aligned}
& v(x)=\int_{H_{\lambda}} P(x, \xi) g(\xi)^{q} d \xi+\int_{H_{\lambda}} P\left(x_{\lambda}, \xi\right) g\left(\xi_{\lambda}\right)^{q} d \xi \\
& g(\xi)=\int_{Q_{\lambda}} P(x, \xi) v(x)^{\frac{q+n}{(n-1) q}} d x+\int_{Q_{\lambda}} P\left(x_{\lambda}, \xi\right) v\left(x_{\lambda}\right)^{\frac{q+n}{(n-1) q}} d x .
\end{aligned}
$$

Case 6.1. $0<q \leq \frac{n}{n-1}$.

In this case, we choose a number $r$ such that

$$
1 \leq r \leq \frac{q+n}{(n-1) q} \text { and } q^{-1}<r
$$

We have

$$
v\left(x_{\lambda}\right)-v(x)=\int_{H_{\lambda}}\left(P(x, \xi)-P\left(x_{\lambda}, \xi\right)\right)\left(g\left(\xi_{\lambda}\right)^{q}-g(\xi)^{q}\right) d \xi
$$

Hence for $x \in \mathcal{B}_{\lambda}^{v}$,

$$
\begin{aligned}
0 & \leq v\left(x_{\lambda}\right)-v(x) \\
& \leq \int_{\mathcal{B}_{\lambda}^{g}}\left(P(x, \xi)-P\left(x_{\lambda}, \xi\right)\right)\left(g\left(\xi_{\lambda}\right)^{q}-g(\xi)^{q}\right) d \xi \\
& \leq q r \int_{\mathcal{B}_{\lambda}^{g}} P(x, \xi) g\left(\xi_{\lambda}\right)^{q-r^{-1}}\left(g\left(\xi_{\lambda}\right)^{1 / r}-g(\xi)^{1 / r}\right) d \xi .
\end{aligned}
$$

It follows that

$$
\begin{aligned}
\left|v_{\lambda}-v\right|_{L^{\frac{n(q+1)}{(n-1) q}\left(\mathcal{B}_{\lambda}^{v}\right)}} & \leq c(n, q, r)\left|g_{\lambda}^{q-r^{-1}}\left(g_{\lambda}^{1 / r}-g^{1 / r}\right)\right|_{L^{\frac{q+1}{q}}\left(\mathcal{B}_{\lambda}^{g}\right)} \\
& \leq c(n, q, r)\left|g_{\lambda}\right|_{L^{q+1}\left(\mathcal{B}_{\lambda}^{g}\right)}^{q-r^{-1}}\left|g_{\lambda}^{1 / r}-g^{1 / r}\right|_{L^{(q+1) r}\left(\mathcal{B}_{\lambda}^{g}\right)}
\end{aligned}
$$

On the other hand, for $\xi \in \mathcal{B}_{\lambda}^{g}$, we have

$$
\begin{aligned}
g\left(\xi_{\lambda}\right) & =\int_{\mathcal{B}_{\lambda}^{v}} P(x, \xi) v\left(x_{\lambda}\right)^{\frac{q+n}{(n-1) q}} d x+\int_{\mathcal{B}_{\lambda}^{v}} P\left(x_{\lambda}, \xi\right) v(x)^{\frac{q+n}{(n-1) q}} d x \\
& +\int_{Q_{\lambda} \backslash \mathcal{B}_{\lambda}^{v}} P(x, \xi) v\left(x_{\lambda}\right)^{\frac{q+n}{(n-1) q}} d x+\int_{Q_{\lambda} \backslash \mathcal{B}_{\lambda}^{v}} P\left(x_{\lambda}, \xi\right) v(x)^{\frac{q+n}{(n-1) q}} d x \\
& \leq \int_{\mathcal{B}_{\lambda}^{v}} P(x, \xi) v\left(x_{\lambda}\right)^{\frac{q+n}{(n-1) q}} d x+\int_{\mathcal{B}_{\lambda}^{v}} P\left(x_{\lambda}, \xi\right) v(x)^{\frac{q+n}{(n-1) q}} d x \\
& +\int_{Q_{\lambda} \backslash \mathcal{B}_{\lambda}^{v}} P(x, \xi) v(x)^{\frac{q+n}{(n-1) q}} d x+\int_{Q_{\lambda} \backslash \mathcal{B}_{\lambda}^{v}} P\left(x_{\lambda}, \xi\right) v\left(x_{\lambda}\right)^{\frac{q+n}{(n-1) q}} d x .
\end{aligned}
$$

Since

$$
\begin{aligned}
g(\xi) & =\int_{\mathcal{B}_{\lambda}^{v}} P(x, \xi) v(x)^{\frac{q+n}{(n-1) q}} d x+\int_{\mathcal{B}_{\lambda}^{v}} P\left(x_{\lambda}, \xi\right) v\left(x_{\lambda}\right)^{\frac{q+n}{(n-1) q}} d x \\
& +\int_{Q_{\lambda} \backslash \mathcal{B}_{\lambda}^{v}} P(x, \xi) v(x)^{\frac{q+n}{(n-1) q}} d x+\int_{Q_{\lambda} \backslash \mathcal{B}_{\lambda}^{v}} P\left(x_{\lambda}, \xi\right) v\left(x_{\lambda}\right)^{\frac{q+n}{(n-1) q}} d x,
\end{aligned}
$$


we see

$$
\begin{aligned}
& g\left(\xi_{\lambda}\right)^{1 / r}-g(\xi)^{1 / r} \\
& \leq\left(\int_{\mathcal{B}_{\lambda}^{v}} P(x, \xi) v\left(x_{\lambda}\right)^{\frac{q+n}{(n-1) q}} d x+\int_{\mathcal{B}_{\lambda}^{v}} P\left(x_{\lambda}, \xi\right) v(x)^{\frac{q+n}{(n-1) q}} d x\right)^{1 / r} \\
& -\left(\int_{\mathcal{B}_{\lambda}^{v}} P(x, \xi) v(x)^{\frac{q+n}{(n-1) q}} d x+\int_{\mathcal{B}_{\lambda}^{v}} P\left(x_{\lambda}, \xi\right) v\left(x_{\lambda}\right)^{\frac{q+n}{(n-1) q}} d x\right)^{1 / r} \\
& =\left(\int_{\mathcal{B}_{\lambda}^{v}}\left|\left(P(x, \xi)^{1 / r} v\left(x_{\lambda}\right)^{\frac{q+n}{(n-1) q r}}, P\left(x_{\lambda}, \xi\right)^{1 / r} v(x)^{\frac{q+n}{(n-1) q r}}\right)\right|_{l^{r}}^{r} d x\right)^{1 / r} \\
& -\left(\int_{\mathcal{B}_{\lambda}^{v}}\left|\left(P(x, \xi)^{1 / r} v(x)^{\frac{q+n}{(n-1) q r}}, P\left(x_{\lambda}, \xi\right)^{1 / r} v\left(x_{\lambda}\right)^{\frac{q+n}{(n-1) q r}}\right)\right|_{l^{r}}^{r} d x\right)^{1 / r} \\
& \leq\left(\int_{\mathcal{B}_{\lambda}^{v}}|(I, I I)|_{l^{r}}^{r} d x\right)^{1 / r} \cdot
\end{aligned}
$$

Here

$$
\begin{gathered}
I=P(x, \xi)^{1 / r}\left(v\left(x_{\lambda}\right)^{\frac{q+n}{(n-1) q r}}-v(x)^{\frac{q+n}{(n-1) q r}}\right) \\
I I=P\left(x_{\lambda}, \xi\right)^{1 / r}\left(v(x)^{\frac{q+n}{(n-1) q r}}-v\left(x_{\lambda}\right)^{\frac{q+n}{(n-1) q r}}\right) .
\end{gathered}
$$

Hence

$$
\begin{aligned}
& g\left(\xi_{\lambda}\right)^{1 / r}-g(\xi)^{1 / r} \\
& \leq 2\left(\int_{\mathcal{B}_{\lambda}^{v}} P(x, \xi)\left(v\left(x_{\lambda}\right)^{\frac{q+n}{(n-1) q r}}-v(x)^{\frac{q+n}{(n-1) q r}}\right)^{r} d x\right)^{1 / r} \\
& \leq \frac{2(q+n)}{(n-1) q r}\left(\int_{\mathcal{B}_{\lambda}^{v}} P(x, \xi) v\left(x_{\lambda}\right)^{\frac{q+n}{(n-1) q}-r}\left(v\left(x_{\lambda}\right)-v(x)\right)^{r} d x\right)^{1 / r} .
\end{aligned}
$$

This implies

$$
\begin{aligned}
& \left|g_{\lambda}^{1 / r}-g^{1 / r}\right| L_{L^{(q+1) r}\left(\mathcal{B}_{\lambda}^{g}\right)} \\
& \leq \frac{2(q+n)}{(n-1) q r}\left|\int_{\mathcal{B}_{\lambda}^{v}} P(x, \xi) v\left(x_{\lambda}\right)^{\frac{q+n}{(n-1) q}-r}\left(v\left(x_{\lambda}\right)-v(x)\right)^{r} d x\right|_{L^{q+1}\left(\mathcal{B}_{\lambda}^{g}\right)}^{1 / r} \\
& \leq c(n, q, r)\left|v_{\lambda}^{\frac{q+n}{(n-1) q}-r}\left(v_{\lambda}-v\right)^{r}\right|_{L^{\frac{n(q+1)}{q+n}}\left(\mathcal{B}_{\lambda}^{v}\right)}^{1 / r} \\
& \leq c(n, q, r)\left|v_{\lambda}\right|_{L^{\frac{n(q) q+1}{(n-1) q}}\left(\mathcal{B}_{\lambda}^{v}\right)}^{\mid \frac{q+n}{(n-1)}}-1 \\
& \quad v_{\lambda}-v_{L^{\frac{n(q+1)}{(n-1) q}}\left(\mathcal{B}_{\lambda}^{v}\right)} .
\end{aligned}
$$

It follows from the two inequalities above that

$$
\left|g_{\lambda}^{1 / r}-g^{1 / r}\right|_{L^{(q+1) r}\left(\mathcal{B}_{\lambda}^{g}\right)} \leq c(n, q, r)|v|_{L^{\frac{n(q+1)}{(n-1) q(}}\left(\mathbb{R}_{+}^{n}\right)}^{\frac{q+n}{(n-1)}-1}|g|_{L^{q+1}\left(2 \lambda e_{1}^{\prime}-\mathcal{B}_{\lambda}^{g}\right)}^{q-r^{-1}}\left|g_{\lambda}^{1 / r}-g^{1 / r}\right|_{L^{(q+1) r}\left(\mathcal{B}_{\lambda}^{g}\right)} .
$$


Here $e_{1}=(1,0, \cdots, 0)$. After these preparations, we will use the method of moving planes to prove the radial symmetry of $g$ and hence $f$.

First, we have to show it is possible to start. Indeed, for $\lambda$ large enough, $|g|_{L^{q+1}\left(2 \lambda e_{1}^{\prime}-\mathcal{B}_{\lambda}^{v}\right)}$ can be arbitrarily small, this implies

$$
\left|g_{\lambda}^{1 / r}-g^{1 / r}\right|_{L^{(q+1) r}\left(\mathcal{B}_{\lambda}^{g}\right)} \leq \frac{1}{2}\left|g_{\lambda}^{1 / r}-g^{1 / r}\right|_{L^{(q+1) r}\left(\mathcal{B}_{\lambda}^{g}\right)}
$$

and hence $\left|g_{\lambda}^{1 / r}-g^{1 / r}\right|_{L^{(q+1) r}\left(\mathcal{B}_{\lambda}^{g}\right)}=0$. It follows that $\mathcal{B}_{\lambda}^{g}=\emptyset$ when $\lambda$ is large enough.

Next we let $\lambda_{0}=\inf \left\{\lambda \in \mathbb{R}: \mathcal{B}_{\lambda^{\prime}}^{g}=\emptyset\right.$ for all $\left.\lambda^{\prime} \geq \lambda\right\}$. It follows from the fact $g(\xi) \rightarrow 0$ as $|\xi| \rightarrow \infty$ and $g(\xi)>0$ for all $\xi \in \mathbb{R}^{n-1}$ that $\lambda_{0}$ must be a finite number. By the definition of $\lambda_{0}$ we know $g_{\lambda_{0}}(\xi) \leq g(\xi)$ for $\xi \in H_{\lambda_{0}}$. We claim that $g_{\lambda_{0}}=g$. Indeed if this is not the case, then since

$$
v_{\lambda_{0}}(x)-v(x)=\int_{H_{\lambda_{0}}}\left(P(x, \xi)-P\left(x_{\lambda_{0}}, \xi\right)\right)\left(g_{\lambda_{0}}(\xi)^{q}-g(\xi)^{q}\right) d \xi
$$

and

$$
g_{\lambda_{0}}(\xi)-g(\xi)=\int_{Q_{\lambda_{0}}}\left(P(x, \xi)-P\left(x_{\lambda_{0}}, \xi\right)\right)\left(v_{\lambda_{0}}(x)^{\frac{q+n}{(n-1) q}}-v(x)^{\frac{q+n}{(n-1) q}}\right) d x
$$

we get $g_{\lambda_{0}}(\xi)<g(\xi)$ for $\xi \in H_{\lambda_{0}}$. It follows that $\chi_{2 \lambda e_{1}^{\prime}-\mathcal{B}_{\lambda}^{g}} \rightarrow 0$ a.e. as $\lambda \uparrow \lambda_{0}$. By dominated convergence theorem we have $|g|_{L^{q+1}\left(2 \lambda e_{1}^{\prime}-\mathcal{B}_{\lambda}^{g}\right)} \rightarrow 0$ as $\lambda \uparrow \lambda_{0}$. It implies

$$
\left|g_{\lambda}^{1 / r}-g^{1 / r}\right|_{L^{(q+1) r}\left(\mathcal{B}_{\lambda}^{g}\right)} \leq \frac{1}{2}\left|g_{\lambda}^{1 / r}-g^{1 / r}\right|_{L^{(q+1) r}\left(\mathcal{B}_{\lambda}^{g}\right)}
$$

when $\lambda$ is very close to $\lambda_{0}$ and hence $\mathcal{B}_{\lambda}^{g}=\emptyset$. This contradicts with the choice of $\lambda_{0}$. Hence when the moving process stops, we must have symmetry. Moreover we claim that $g_{\lambda}(\xi)<g(\xi)$ for $\xi \in H_{\lambda}$ when $\lambda>\lambda_{0}$. Indeed for any $\lambda>\lambda_{0}$ we can not have $g_{\lambda}=g$ because otherwise $g$ is periodic in the first direction and can not lie in $L^{q+1}\left(\mathbb{R}^{n-1}\right)$. Hence $g_{\lambda}<g$ in $H_{\lambda}$.

By translation, we may assume $g(0)=\max _{\xi \in \mathbb{R}^{n-1}} g(\xi)$, then it follows that the moving plane process from any direction must stop at the origin. Hence $g$ must be radial symmetric and strictly decreasing in the radial direction.

Case 6.2. $\frac{n}{n-1} \leq q<\infty$.

In this case, we choose a number $r$ such that

$$
1 \leq r<q \text { and } \frac{(n-1) q}{q+n} \leq r .
$$

We have

$$
g\left(\xi_{\lambda}\right)-g(\xi)=\int_{Q_{\lambda}}\left(P(x, \xi)-P\left(x_{\lambda}, \xi\right)\right)\left(v\left(x_{\lambda}\right)^{\frac{q+n}{(n-1) q}}-v(x)^{\frac{q+n}{(n-1) q}}\right) d x .
$$


Hence for $\xi \in \mathcal{B}_{\lambda}^{g}$,

$$
\begin{aligned}
0 & \leq g\left(\xi_{\lambda}\right)-g(\xi) \\
& \leq \int_{\mathcal{B}_{\lambda}^{v}}\left(P(x, \xi)-P\left(x, \xi_{\lambda}\right)\right)\left(v\left(x_{\lambda}\right)^{\frac{q+n}{(n-1) q}}-v(x)^{\frac{q+n}{(n-1) q}}\right) d x \\
& \leq \frac{(q+n) r}{(n-1) q} \int_{\mathcal{B}_{\lambda}^{v}} P(x, \xi) v\left(x_{\lambda}\right)^{\frac{q+n}{(n-1) q}-r^{-1}}\left(v\left(x_{\lambda}\right)^{1 / r}-v(x)^{1 / r}\right) d x .
\end{aligned}
$$

It follows that

$$
\begin{aligned}
& \left|g_{\lambda}-g\right|_{L^{q+1}\left(\mathcal{B}_{\lambda}^{g}\right)} \\
& \leq c(n, q, r)\left|v_{\lambda}^{\frac{q+n}{(n-1) q}-r^{-1}}\left(v_{\lambda}^{1 / r}-v^{1 / r}\right)\right|_{L^{\frac{n(q+1)}{q+n}}\left(\mathcal{B}_{\lambda}^{v}\right)} \\
& =c(n, q, r)\left|v_{\lambda}\right|_{L^{\frac{n(q+1)}{(n-1) q}}\left(\mathcal{B}_{\lambda}^{v}\right)}^{\frac{q+n}{(n-1)}-r^{-1}}\left|v_{\lambda}^{1 / r}-v^{1 / r}\right|_{L^{\frac{n(q+1) r}{(n-1) q}}\left(\mathcal{B}_{\lambda}^{v}\right)} .
\end{aligned}
$$

On the other hand, for $x \in \mathcal{B}_{\lambda}^{v}$, we have

$$
\begin{aligned}
v\left(x_{\lambda}\right) & \leq \int_{\mathcal{B}_{\lambda}^{g}} P(x, \xi) g\left(\xi_{\lambda}\right)^{q} d \xi+\int_{\mathcal{B}_{\lambda}^{g}} P\left(x_{\lambda}, \xi\right) g(\xi)^{q} d \xi \\
& +\int_{H_{\lambda} \backslash \mathcal{B}_{\lambda}^{g}} P(x, \xi) g(\xi)^{q} d \xi+\int_{H_{\lambda} \backslash \mathcal{B}_{\lambda}^{g}} P\left(x_{\lambda}, \xi\right) g\left(\xi_{\lambda}\right)^{q} d \xi .
\end{aligned}
$$

Since

$$
\begin{aligned}
v(x) & =\int_{\mathcal{B}_{\lambda}^{g}} P(x, \xi) g(\xi)^{q} d \xi+\int_{\mathcal{B}_{\lambda}^{g}} P\left(x_{\lambda}, \xi\right) g\left(\xi_{\lambda}\right)^{q} d \xi \\
& +\int_{H_{\lambda} \backslash \mathcal{B}_{\lambda}^{g}} P(x, \xi) g(\xi)^{q} d \xi+\int_{H_{\lambda} \backslash \mathcal{B}_{\lambda}^{g}} P\left(x_{\lambda}, \xi\right) g\left(\xi_{\lambda}\right)^{q} d \xi
\end{aligned}
$$

we see

$$
\begin{aligned}
0 & \leq v\left(x_{\lambda}\right)^{1 / r}-v(x)^{1 / r} \\
& \leq\left(\int_{\mathcal{B}_{\lambda}^{g}} P(x, \xi) g\left(\xi_{\lambda}\right)^{q} d \xi+\int_{\mathcal{B}_{\lambda}^{g}} P\left(x_{\lambda}, \xi\right) g(\xi)^{q} d \xi\right)^{1 / r} \\
& -\left(\int_{\mathcal{B}_{\lambda}^{g}} P(x, \xi) g(\xi)^{q} d \xi+\int_{\mathcal{B}_{\lambda}^{g}} P\left(x_{\lambda}, \xi\right) g\left(\xi_{\lambda}\right)^{q} d \xi\right)^{1 / r} \\
& \leq\left(\int_{\mathcal{B}_{\lambda}^{g}}\left|\left(P(x, \xi)^{1 / r}\left(g\left(\xi_{\lambda}\right)^{q / r}-g(\xi)^{q / r}\right), P\left(x_{\lambda}, \xi\right)^{1 / r}\left(g(\xi)^{q / r}-g\left(\xi_{\lambda}\right)^{q / r}\right)\right)\right|_{l^{r}}^{r} d \xi\right)^{1 / r} \\
& \leq 2\left(\int_{\mathcal{B}_{\lambda}^{g}} P(x, \xi)\left(g\left(\xi_{\lambda}\right)^{q / r}-g(\xi)^{q / r}\right)^{r} d \xi\right)^{1 / r} \\
& \leq \frac{2 q}{r}\left(\int_{\mathcal{B}_{\lambda}^{g}} P(x, \xi) g\left(\xi_{\lambda}\right)^{q-r}\left(g\left(\xi_{\lambda}\right)-g(\xi)\right)^{r} d \xi\right)^{1 / r} .
\end{aligned}
$$


Hence

$$
\begin{aligned}
& \left|v_{\lambda}^{1 / r}-v^{1 / r}\right|_{L^{\frac{n(q+1) r}{(n-1) q}}}\left(\mathcal{B}_{\lambda}^{v}\right) \\
& \leq \frac{2 q}{r}\left|\int_{\mathcal{B}_{\lambda}^{g}} P(x, \xi) g\left(\xi_{\lambda}\right)^{q-r}\left(g\left(\xi_{\lambda}\right)-g(\xi)\right)^{r} d \xi\right|_{L^{\frac{n(q+1)}{(n-1) q}}\left(\mathcal{B}_{\lambda}^{v}\right)}^{1 / r} \\
& \leq c(n, q, r)\left|g_{\lambda}^{q-r}\left(g_{\lambda}-g\right)^{r}\right|_{L^{\frac{q+1}{q}}}^{1 / r}\left(\mathcal{B}_{\lambda}^{g}\right) \\
& \leq c(n, q, r)\left|g_{\lambda}\right|_{L^{q+1}\left(\mathcal{B}_{\lambda}^{g}\right)}^{\frac{q-r}{r}}\left|g_{\lambda}-g\right|_{L^{q+1}\left(\mathcal{B}_{\lambda}^{g}\right)} .
\end{aligned}
$$

Combining the two inequalities together we see

$$
\left|g_{\lambda}-g\right|_{L^{q+1}\left(\mathcal{B}_{\lambda}^{g}\right)} \leq c(n, q, r)|v|_{L^{\frac{n(q+1)}{(n-1) q}\left(\mathbb{R}_{+}^{n}\right)}}^{\frac{q+n}{(n-1) q}-r^{-1}}|g|_{L^{q+1}\left(2 \lambda e_{1}^{\prime}-\mathcal{B}_{\lambda}^{g}\right)}^{\frac{q-r}{r}}\left|g_{\lambda}-g\right|_{L^{q+1}\left(\mathcal{B}_{\lambda}^{g}\right)} .
$$

With this inequality at hand, we may proceed in the same way as in the Case 6.1 to get the conclusion that $g$ is radial symmetric with respect to some point and strictly decreasing along the radial direction.

Next we look at the special power $p=\frac{2(n-1)}{n-2}$.

Proof of Proposition 6.1. If we know $f \in L^{\frac{2(n-1)}{n-2}}\left(\mathbb{R}^{n-1}\right)$, then it follows from Theorem 6.1 that $f \in C^{\infty}\left(\mathbb{R}^{n-1}\right)$, it is strictly positive and radial symmetric with respect to some point. By translation we may assume $f$ is radial symmetric with respect to 0 .

On the other hand, if $f$ is a solution to the equation, let $u(x)=(P f)(x)$, $\tilde{f}(\xi)=\frac{1}{|\xi|^{n-2}} f\left(\frac{\xi}{|\xi|^{2}}\right)$ and $\widetilde{u}(x)=\frac{1}{|x|^{n-2}} u\left(\frac{x}{|x|^{2}}\right)$, by change of variable we know

$$
\widetilde{u}(x)=(P \widetilde{f})(x), \quad \widetilde{f}(\xi)^{\frac{n}{n-2}}=\int_{\mathbb{R}_{+}^{n}} P(x, \xi) \widetilde{u}(x)^{\frac{n+2}{n-2}} d x
$$

and $|\tilde{f}|_{L^{\frac{2(n-1)}{n-2}}\left(\mathbb{R}^{n-1}\right)}=|f|_{L^{\frac{2(n-1)}{n-2}}\left(\mathbb{R}^{n-1}\right)}$. In particular, $\tilde{f} \in L^{\frac{2(n-1)}{n-2}}$ and satisfies the same equation.

Let $e_{1}=(1,0, \cdots, 0) \in \mathbb{R}^{n}$, then it follows from Theorem 6.1 that $f_{1}(\xi)=$ $\frac{1}{|\xi|^{n-2}} f\left(\frac{\xi}{|\xi|^{2}}-e_{1}^{\prime}\right)$ is smooth and radial symmetric with respect to some point. It follows from Proposition 4.1 and the fact that $f \in L^{\frac{2(n-1)}{n-2}}\left(\mathbb{R}^{n-1}\right)$ that for some $c_{1}>0$ and $c_{2}>0, f(\xi)=\left(c_{1}|\xi|^{2}+c_{2}\right)^{-\frac{n-2}{2}}$. Since $f$ satisfies the equation, it follows that for some $\lambda>0, f(\xi)=c(n)\left(\frac{\lambda}{\lambda^{2}+|\xi|^{2}}\right)^{\frac{n-2}{2}}$.

Next we want to show under the assumption of the Proposition 6.1, $f$ always lies in $L^{\frac{2(n-1)}{n-2}}\left(\mathbb{R}^{n-1}\right)$. This will be proved by contradiction. Indeed, if this is not the case, then $\int_{\mathbb{R}^{n-1}} f(\xi)^{\frac{2(n-1)}{n-2}} d \xi=\infty$. Let $g_{0}(\xi)=f(\xi)^{\frac{n}{n-2}}, v_{0}(x)=(P f)(x)$, then $g_{0} \in L_{l o c}^{\frac{2(n-1)}{n}}\left(\mathbb{R}^{n-1}\right), \int_{\mathbb{R}^{n-1}} g_{0}(\xi)^{\frac{2(n-1)}{n}} d \xi=\infty$ and

$$
v_{0}(x)=\int_{\mathbb{R}^{n-1}} P(x, \xi) g_{0}(\xi)^{\frac{n-2}{n}} d \xi, \quad g_{0}(\xi)=\int_{\mathbb{R}_{+}^{n}} P(x, \xi) v_{0}(x)^{\frac{n+2}{n-2}} d x .
$$


It follows from the proof of Theorem 5.1 that $g_{0} \in C^{\infty}\left(\mathbb{R}^{n-1}\right)$ and $v_{0} \in C^{\infty}\left(\overline{\mathbb{R}}_{+}^{n}\right)$.

Let $g(\xi)=\frac{1}{|\xi|^{n}} g_{0}\left(\frac{\xi}{|\xi|^{2}}\right), v(x)=\frac{1}{|x|^{n-2}} v_{0}\left(\frac{x}{|x|^{2}}\right)$, then

$$
v(x)=\int_{\mathbb{R}^{n-1}} P(x, \xi) g(\xi)^{\frac{n-2}{n}} d \xi, \quad g(\xi)=\int_{\mathbb{R}_{+}^{n}} P(x, \xi) v(x)^{\frac{n+2}{n-2}} d x .
$$

Moreover for any $R>0, \int_{\mathbb{R}^{n-1} \backslash B_{R}} g(\xi)^{\frac{2(n-1)}{n}} d \xi<\infty$ and $\int_{\mathbb{R}^{n-1}} g(\xi)^{\frac{2(n-1)}{n}} d \xi=\infty$. For $\lambda>0$, we define $H_{\lambda}, g_{\lambda}$ as in the Case 6.1 of the proof of Theorem6.1 but let $\mathcal{B}_{\lambda}^{g}=\left\{\xi \in H_{\lambda} \backslash\{0\}: g_{\lambda}(\xi)>g(\xi)\right\}$. Put the number in the proof of Theorem 6.1 $r=\frac{n+2}{n-2}$, then the same argument shows

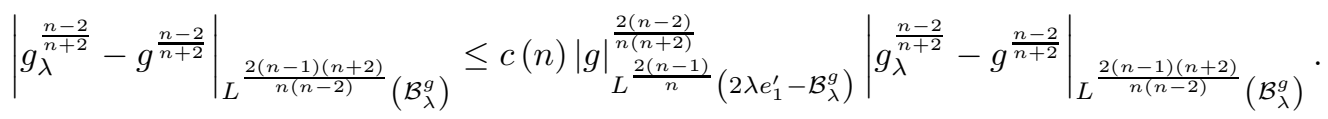

Note that for $\xi \in \mathcal{B}_{\lambda}^{g}, g_{\lambda}(\xi)>g(\xi)$, hence

$$
\int_{\mathcal{B}_{\lambda}^{g}} g(\xi)^{\frac{2(n-1)}{n}} d \xi \leq \int_{\mathcal{B}_{\lambda}^{g}} g_{\lambda}(\xi)^{\frac{2(n-1)}{n}} d \xi \leq \int_{\mathbb{R}^{n-1} \backslash H_{\lambda}} g(\xi)^{\frac{2(n-1)}{n}} d \xi<\infty .
$$

When $\lambda$ is large enough, it implies

$$
\left|g_{\lambda}^{\frac{n-2}{n+2}}-g^{\frac{n-2}{n+2}}\right|_{L}^{\frac{2(n-1)(n+2)}{n(n-2)}\left(\mathcal{B}_{\lambda}^{g}\right)} \leq \frac{1}{2}\left|g^{\frac{n-2}{n+2}}-g^{\frac{n-2}{n+2}}\right|_{L}^{\frac{2(n-1)(n+2)}{n(n-2)}}\left(\mathcal{B}_{\lambda}^{g}\right)
$$

and hence $\left|g_{\lambda}^{\frac{n-2}{n+2}}-g^{\frac{n-2}{n+2}}\right|_{L}^{\frac{2(n-1)(n+2)}{n(n-2)}\left(\mathcal{B}_{\lambda}^{g}\right)}=0, \mathcal{B}_{\lambda}^{g}=\emptyset$. Let

$$
\lambda_{0}=\inf \left\{\lambda>0: \mathcal{B}_{\lambda^{\prime}}^{g}=\emptyset \text { for all } \lambda^{\prime} \geq \lambda\right\} .
$$

We claim $\lambda_{0}=0$. Indeed if this is not the case, then $\lambda_{0}>0$. We may argue as in the Case 6.1 of the proof of Theorem 6.1 and get $g_{\lambda_{0}}=g$. In particular, this would imply $\int_{\mathbb{R}^{n-1}} g(\xi)^{\frac{2(n-1)}{n}} d \xi<\infty$, a contradiction. It follows that $\lambda_{0}=0$ and $g\left(\xi_{1}, \xi^{\prime \prime}\right) \geq g\left(-\xi_{1}, \xi^{\prime \prime}\right)$ for $\xi_{1}<0$. Since we may perform this process along any direction, we see $g$ must be radial symmetric with respect to 0 . Hence $g_{0}$ must be radial symmetric with respect to 0 . For any $\zeta \in \mathbb{R}^{n-1}$, we may apply the argument to $g_{0}(\cdot+\zeta)$ and deduce that $g_{0}$ is also radial symmetric with respect to $\zeta$, hence $g_{0}$ must be a constant function, so is $f$. But this contradicts with the fact that $f$ satisfies the equation.

\section{REFERENCES}

[C] T. Carleman. Zur Theorie de Minimalfächen. Math Z. 9 (1921), 154-160.

[CGS] L. A. Caffarelli, B. Gidas and J. Spruck. Asymptotic symmetry and local behavior of semilinear elliptic equations with critical Sobolev growth. Comm Pure Appl Math. 42 (1989), no. 3, 271-297.

[CL] E. A. Carlen and M. Loss. Extremals of functionals with competing symmetries. J Funct Anal. 88 (1990), no. 2, 437-456.

[ChL] W. X. Chen and C. M. Li. Regularity of solutions for a system of integral equations. Comm Pure Appl Anal, 4(1), (2005), 1-8.

[CLO1] W. X. Chen, C. M. Li and B. Ou. Classification of solutions for a system of integral equations. Comm in Partial Differential Equations, 30 (2005), 59-65.

[CLO2] W. X. Chen, C. M. Li and B. Ou. Classification of solutions for an integral equation. Comm Pure Appl Math, 59 (2006), no. 3, 330-343. 
[CLO3] W. X. Chen, C. M. Li and B. Ou. Alternative proofs on the radial symmetry and monotonicity of positive regular solutions to a singular integral equation. IMA Preprint Series \#2044.

[EG] L. C. Evans and R. F. Gariepy. Measure theory and fine properties of functions. Studies in Advanced Mathematics. CRC Press, Boca Raton, FL, 1992.

[GNN] B. Gidas, W. M. Ni and L. Nirenberg. Symmetry and related properties via the maximum principle. Communications in Mathematical Physics. 68 (1979), 209-243.

[GT] D. Gilbarg and N. S. Trudinger. Elliptic partial differential equations of second order. 2nd edition, 3rd printing. Berlin: Springer-Verlag, 1998.

[HL] Q. Han and F. H. Lin. Elliptic partial differential equations. Courant Lecture Notes, volume 1. American Mathematical Society, 2000.

[Hn] F. B. Hang. On the integral systems related to Hardy-Littlewood-Sobolev inequality. Preprint, 2005.

[HWY] F. B. Hang, X. D. Wang and X. D. Yan. An integral equation in conformal geometry. In preparation.

[L] Y. Y. Li. Remark on some conformally invariant integral equations: the method of moving spheres. Journal of European Mathematical Society. 6 (2004), 153-180.

[LZ] Y. Li and M. Zhu. Uniqueness theorems through the method of moving spheres. Duke Mathematical Journal. 80 no. 2 (1995), 383-417.

[Li1] E. H. Lieb. Existence and uniqueness of the minimizing solution of Choquard's nonlinear equation. Studies in Appl Math. 57 (1977), 93-105.

[Li2] E. H. Lieb. Sharp constants in the Hardy-Littlewood-Sobolev and related inequalities. Ann of Math, 118(2), (1983), 349-374.

[LiL] E. H. Lieb and M. Loss. Analysis. Second edition. Graduate Studies in Mathematics, 14. American Mathematical Society, Providence, RI, 2001.

[Lion] P. L. Lions. The concentration-compactness principle in the calculus of variations. The limit case. II. Rev. Mat. Iberoamericana 1 (1985), no. 2, 45-121.

[O] B. Ou. A remark on a singular integral equation. Houston J Math. 25 (1999), 181-184.

[S] E. M. Stein. Singular integrals and differentiability properties of functions. Princeton University Press, Princeton, New Jersey, 1970.

[SW] E. M. Stein and G. Weiss. Introduction to Fourier analysis on Euclidean spaces. Princeton Mathematical Series, No. 32. Princeton University Press, Princeton NJ, 1971.

Department of Mathematics, Princeton University, Fine Hall, Washington Road, PRINCETON, NJ 08544

E-mail address: fhang@math.princeton.edu

Department of Mathematics, Michigan State University, East Lansing, Mi 48824

E-mail address: xwang@math.msu.edu

Department of Mathematics, Michigan State University, East Lansing, Mi 48824

E-mail address: xiayan@math.msu.edu 